Article

\title{
Lineage, Antimicrobial Resistance and Virulence of Citrobacter spp
}

\author{
Liyun Liu ${ }^{1, *, \dagger}$, Liyun Qin ${ }^{2, \dagger}$, Shuai Hao ${ }^{3,+}$, Ruiting Lan ${ }^{4,+}$, Baohong $\mathrm{Xu}^{2}$, Yumei Guo ${ }^{2}$, \\ Ruiping Jiang ${ }^{2}$, Hui Sun ${ }^{1}$, Xiaoping Chen ${ }^{1}$, Xinchao $\mathrm{LV}^{2}$, Jianguo $\mathrm{Xu}^{1}$ and Chuan Zhao ${ }^{2, *}$
}

1 State Key Laboratory of Infectious Disease Prevention and Control, National Institute for Communicable Disease Control and Prevention, Chinese Center for Disease Control and Prevention, Beijing 102206, China; sunhui@icdc.cn (H.S.); chenxiaoping@icdc.cn (X.C.); xujianguo@icdc.cn (J.X.)

2 Shijiazhuang Center for Disease Control and Prevention, Shijiazhuang 050011, China; qinliyun-2007@163.com (L.Q.); Wsws1120@163.com (B.X.); Guokexin2199@163.com (Y.G.); Jrpok97@163.com (R.J.); lvxinzhao1986@163.com (X.L.)

3 Beijing Advanced Innovation Center for Food Nutrition and Human Health, Beijing Engineering and Technology Research Center of Food Additives, Beijing Technology and Business University, Beijing 100048, China; haoshuai@btbu.edu.cn

4 School of Biotechnology and Biomolecular Sciences, University of New South Wales, Sydney, NSW 2052, Australia; r.lan@unsw.edu.au

* Correspondence: liuliyun@icdc.cn (L.L.); shijiazhuangcdc@sina.com (C.Z.)

+ These authors contributed equally to this work.

Received: 4 February 2020; Accepted: 4 March 2020; Published: 6 March 2020

check for updates

\begin{abstract}
Citrobacter spp. are opportunistic human pathogens which can cause nosocomial infections, sporadic infections and outbreaks. In order to determine the genetic diversity, in vitro virulence properties and antimicrobial resistance profiles of Citrobacter spp., 128 Citrobacter isolates obtained from human diarrheal patients, foods and environment were assessed by multilocus sequence typing (MLST), antimicrobial susceptibility testing and adhesion and cytotoxicity testing to HEp-2 cells. The 128 Citrobacter isolates were typed into 123 sequence types (STs) of which 101 were novel STs, and these STs were divided into five lineages. Lineages I and II contained C. freundii isolates; Lineage III contained all C. braakii isolates, while Lineage IV and V contained C. youngae isolates. Lineages II and $\mathrm{V}$ contained more adhesive and cytotoxic isolates than Lineages I, III, and IV. Fifty-one of the 128 isolates were found to be multidrug-resistant (MDR, $\geq 3$ ) and mainly distributed in Lineages I, II, and III. The prevalence of quinolone resistance varied with Lineage III (C. braakii) having the highest proportion of resistant isolates (52.6\%), followed by Lineage I (C. freundii) with $23.7 \%$. Seven $q n r B$ variants, including two new alleles ( $q n r B 93$ and qnrB94) were found with Lineage I being the main reservoir. In summary, highly cytotoxic MDR isolates from diarrheal patients may increase the risk of severe disease.
\end{abstract}

Keywords: Citrobacter; sequence types; multidrug resistance; adhesion; cytotoxicity

\section{Background}

The genus Citrobacter contained 11 species, most of which are opportunistic human pathogens that can cause nosocomial infections [1], and a range of other infections [2-6]. In this study, we focus on three Citrobacter species, C. freundii, C. youngae and C. braakii, as potential foodborne pathogens. C. freundii is the most commonly isolated Citrobacter species causing diarrhea and other infections $[7,8]$, while $C$. youngae and C. braakii are rarely reported to cause infections. Some $C$. freundii strains caused food poisoning or diarrhea in humans which were found to carry virulent factors, such as Shiga-like toxins, heat-stable toxins, or virulence islands $[9,10]$. C. youngae can cause peritonitis [11]. C. braakii has 
been isolated from the peritonea of acute peritonitis patients, as well as from food products [12-15]. In our previous studies, C. freundii and C. youngae have been isolated from the fecal samples of diarrheal patients and different types of food samples, and are potential foodborne pathogens [10,16], while C. braakii has been isolated from food [10].

Antibiotic resistance of Citrobacter has increased, and multidrug-resistant (MDR) isolates have frequently been reported [10,17-21]. Frequent isolation of MDR C. freundii with resistance to $\beta$-lactams, quinolones and aminoglycosides has been reported by several international surveillance programs [18]. In our previous study, $31.7 \%$ of $C$. freundii isolates were MDR that were resistant to $\beta$-lactams, quinolones, aminoglycosides, tetracyclines, phenicols, sulfonamides or nitrofuran $[10,16]$. Furthermore, although not MDR, 4.9\% were also resistant to aminoglycosides, $\beta$-lactams, and quinolones $[10,16]$.

Antibiotic resistant Citrobacter often harbored extended-spectrum $\beta$-lactamase (ESBL) $[1,19,20]$, and plasmid-mediated quinolone resistance (PMQR) determinants [21]. The prevalence of ESBL and PMQR Citrobacter isolates was reported from several international studies [1,19-21]. In our previous study, we identified four $C$. freundii isolates from clinical sources and foods that were ESBL producing and 21 isolates from clinical sources and foods that harbored PMQR genes, including $a a c\left(6^{\prime}\right)-I b-c r$, qnrS1, qnrB9, qnrB13, qnrB16, qnrB17, qnrB63, qnrB76, qnrB77, or qnrB92 [10,16].

Fluoroquinolone resistance is associated with mutations in DNA gyrase and topoisomerase IV genes, in particular, mutations in the quinolone resistance-determining regions (QRDRs) of $g y r A$ and parC genes [22]. It has been reported that $C$. freundii isolates with reduced susceptibility to fluoroquinolones were found to contain Thr83Ile or Asp87Asn mutation in gyrA [22,23]. In our previous study, we screened mutations in the QRDRs of gyr A and parC genes in fluoroquinolone resistant isolates. Four of the six fluoroquinolone resistant isolates were found to carry Thr59Ile, Gln111Arg and Ile134Val mutations of the gyrA gene [16]. However, it remains to be determined whether these mutations confer resistance to fluoroquinolones. No mutations in the QRDR region of the parC gene was found in the six fluoroquinolones resistant isolates [16].

To further understand the genetic diversity, virulence and antibiotic resistance of Citrobacter spp. From different sources, in this study, we isolated 128 Citrobacter isolates from diarrheal outpatients, food and environment in Shijiazhuang Hebei Province, China. We performed multilocus sequence typing (MLST) to determine the relationships of the isolates and screened for $b l a_{\mathrm{CTX}-\mathrm{M}}, b l a_{\mathrm{SHV}}, b l a_{\mathrm{TEM}}$ and $q n r$ genes by PCR and mutations in gyrA and parC genes by PCR sequencing and assayed the adhesion and cytotoxicity to Hep-2 cells of all isolates.

\section{Results}

\subsection{Multilocus Sequence Typing of Citrobacter Isolates}

The 128 Citrobacter isolates were typed into 123 STs with the 67 C. freundii isolates dividing into 65 STs, the 45 C. braakii isolates into 42 STs and the 16 C. youngae isolates into 16 STs (Table 1 and Figure 1). Of the 123 STs, 101 were novel STs (from ST269 to ST387). No STs were predominant. Two STs (ST1 and ST100) of C. freundii each contained two isolates. ST1 contained one isolate from a diarrheal patient and one from food. ST100 contained one isolate from the environment and one from food. ST357 and ST375 of C. braakii each contained two isolates from diarrheal patients or foods. ST297 of C. braakii contained two isolates, with one from the environment and one from food.

The concatenated sequence of the seven housekeeping genes for the 128 Citrobacter isolates, was used to construct a neighbor-joining tree (Figure 1). Salmonella LT2 was used as an outgroup. The 128 Citrobacter isolates were divided into five lineages with strong bootstrap support to the lineage divisions. Lineages I and II contained C. freundii isolates, while Lineages IV and V contained C. youngae isolates exclusively. Lineage III contained all C. braakii isolates. Citrobacter isolates from different sources were not grouped by source and were dispersed among different lineages (Table 1 and Figure 1). 
Table 1. Adherence, cytotoxicity, multidrug-resistant and Genotypes of 128 Citrobacter Isolates.

\begin{tabular}{|c|c|c|c|c|c|c|c|c|c|}
\hline $\begin{array}{l}\text { Clusters and } \\
\text { Species }\end{array}$ & Isolates & Year & Source & STs & Adhesion & LDH & NDR & ESBLs & $q n r$ \\
\hline Lineage I & HB2016004 & 2016 & $\mathrm{D}$ & 17 & $* * *$ & $12.4 \pm 0.4$ & 2 & \multirow{6}{*}{$\begin{array}{c}\text { bla }_{\text {CTX-M-3 }} \\
\text { bla }_{\mathrm{TEM}-1}\end{array}$} & $q n r B 9$ \\
\hline \multirow[t]{28}{*}{ C. freundii } & HB2016019 & 2016 & F & 284 & $+/-$ & $6.3 \pm 2.2$ & 9 & & $q n r B 9$ \\
\hline & HB2016023 & 2016 & F & 288 & * & $8.9 \pm 0.2$ & 9 & & $a a c\left(6^{\prime}\right)-I b-c r, q n r B 9$ \\
\hline & HB2016024 & 2016 & F & 289 & * & $20.5 \pm 1.3$ & 3 & & \\
\hline & HB2016034 & 2016 & F & 260 & * & $5.0 \pm 1.4$ & 7 & & qnrS1 \\
\hline & HB2016036 & 2016 & D & 298 & $+/-$ & $0.6 \pm 0.2$ & 2 & & \\
\hline & HB2017002 & 2017 & $\mathrm{D}$ & 300 & $+/-$ & $10.3 \pm 1.8$ & 1 & \multirow[t]{3}{*}{$b l a_{\mathrm{CTX}-\mathrm{M}-9}$} & $\begin{array}{l}\text { qnrS1,qnrB13 } \\
\text { include the LexA } \\
\text { binding site }\end{array}$ \\
\hline & HB2017003 & 2017 & D & 301 & * & $8.9 \pm 0.8$ & 2 & & \\
\hline & HB2017004 & 2017 & $\mathrm{D}$ & 85 & * & $12.3 \pm 2.2$ & 2 & & \\
\hline & HB2017006 & 2017 & $\mathrm{D}$ & 303 & ** & $27.3 \pm 0.6$ & 2 & & $\begin{array}{c}\text { qnrB76 include } \\
\text { the LexA binding } \\
\text { site }\end{array}$ \\
\hline & HB2017009 & 2017 & $\mathrm{D}$ & 304 & * & $11.9 \pm 0.6$ & 4 & & $\begin{array}{l}\text { the LexA binding } \\
\text { site }\end{array}$ \\
\hline & HB2017012 & 2017 & $\mathrm{D}$ & 306 & * & $7.1 \pm 0.4$ & 1 & & $\begin{array}{l}\text { qnrB76 include } \\
\text { the LexA binding } \\
\text { site }\end{array}$ \\
\hline & HB2017016 & 2017 & $\mathrm{D}$ & 309 & * & $15.0 \pm 1.7$ & 1 & & \\
\hline & HB2017017 & 2017 & D & 1 & * & $15.9 \pm 1.0$ & 2 & & \\
\hline & HB2017026 & 2017 & $\mathrm{D}$ & 313 & $* *$ & $17.4 \pm 1.1$ & 6 & & $a a c\left(6^{\prime}\right)-I b-c r$ \\
\hline & HB2017031 & 2017 & D & 318 & $+/-$ & $12.7 \pm 0.2$ & 2 & & qnrB9 \\
\hline & HB2017033 & 2017 & E & 320 & $* *$ & $19.1 \pm 3.2$ & 0 & & qnrB76 \\
\hline & HB2017036 & 2017 & E & 322 & $* *$ & $14.1 \pm 0.8$ & 3 & & \\
\hline & HB2017038 & 2017 & F & 324 & * & $12.4 \pm 8.9$ & 4 & & qnrB94 \\
\hline & HB2017039 & 2017 & F & 325 & * & $19.8 \pm 5.7$ & 2 & & qnrB17 \\
\hline & HB2017040 & 2017 & F & 326 & * & $6.7 \pm 1.4$ & 8 & & \\
\hline & HB2017042 & 2017 & $\mathrm{~F}$ & 328 & - & $8.9 \pm 7.8$ & 9 & \multirow{5}{*}{$\begin{array}{c}\text { bla }_{\mathrm{TEM}-1} \\
b l a_{\mathrm{CTX}-\mathrm{M}-9} \\
b l a_{\mathrm{TEM}-1}\end{array}$} & \\
\hline & HB2017045 & 2017 & F & 331 & * & $14.3 \pm 1.8$ & 7 & & qnrS1 \\
\hline & HB2017052 & 2017 & $\mathrm{~F}$ & 337 & $* *$ & $15.5 \pm 1.5$ & 2 & & \\
\hline & HB2017053 & 2017 & F & 338 & $* *$ & $21.6 \pm 2.5$ & 4 & & qnrB9 \\
\hline & HB2017054 & 2017 & $\mathrm{~F}$ & 339 & $* * *$ & $16.5 \pm 3.6$ & 2 & & $q n r B 76$ \\
\hline & HB2017059 & 2017 & $\mathrm{~F}$ & 343 & * & $12.8 \pm 0.4$ & 7 & \multirow{3}{*}{$\begin{array}{c}\text { bla }_{\text {CTX-M-3' }} \\
\text { bla }_{\text {CTX-M-9 }} \\
\text { bla }_{\text {CTX-M-9, }} \\
\text { bla }_{\text {TEM-1 }}\end{array}$} & qnrB93 \\
\hline & HB2017060 & 2017 & F & 344 & $+/-$ & $11.9 \pm 1.6$ & 7 & & \\
\hline & HB2017061 & 2017 & $\mathrm{~F}$ & 1 & $* *$ & $17.3 \pm 2.4$ & 2 & & \\
\hline Lineage II & HB2016001 & 2016 & $\mathrm{D}$ & 269 & ** & $11.0 \pm 2.1$ & 1 & \multirow{28}{*}{$b l a_{\mathrm{TEM}-1}$} & \multirow{28}{*}{$a a c\left(6^{\prime}\right)-I b-c r, q n r B 2$} \\
\hline \multirow[t]{27}{*}{ C. freundii } & HB2016002 & 2016 & $\mathrm{D}$ & 216 & * & $10.4 \pm 0.2$ & 3 & & \\
\hline & HB2016003 & 2016 & D & 270 & $* *$ & $15.6 \pm 1.1$ & 1 & & \\
\hline & HB2016006 & 2016 & E & 272 & $* *$ & $9.3 \pm 2.8$ & 1 & & \\
\hline & HB2016008 & 2016 & F & 274 & *** & $22.7 \pm 7.3$ & 8 & & \\
\hline & HB2016010 & 2016 & E & 276 & * & $8.4 \pm 1.9$ & 1 & & \\
\hline & HB2016011 & 2016 & F & 100 & $* * *$ & $22.6 \pm 3.0$ & 1 & & \\
\hline & HB2016012 & 2016 & F & 277 & $* *$ & $16.9 \pm 1.5$ & 2 & & \\
\hline & HB2016013 & 2016 & F & 278 & $* *$ & $15.3 \pm 3.9$ & 3 & & \\
\hline & HB2016017 & 2016 & F & 282 & $* * *$ & $21.4 \pm 7.3$ & 1 & & \\
\hline & HB2016018 & 2016 & F & 283 & $+/-$ & $9.2 \pm 1.5$ & 2 & & \\
\hline & HB2017001 & 2017 & D & 169 & $* * *$ & $29.4 \pm 5.8$ & 2 & & \\
\hline & HB2017008 & 2017 & D & 12 & $*$ & $13.6 \pm 0.7$ & 5 & & \\
\hline & HB2017011 & 2017 & D & 163 & $* *$ & $15.8 \pm 0.7$ & 7 & & \\
\hline & HB2017013 & 2017 & D & 307 & $* *$ & $6.8 \pm 0.3$ & 1 & & \\
\hline & HB2017014 & 2017 & D & 308 & ** & $18.0 \pm 13.5$ & 1 & & \\
\hline & HB2017018 & 2017 & $\mathrm{D}$ & 125 & ** & $21.5 \pm 7.3$ & 2 & & \\
\hline & HB2017019 & 2017 & $\mathrm{D}$ & 217 & $* *$ & $14.6 \pm 1.4$ & 1 & & \\
\hline & HB2017020 & 2017 & D & 310 & $* * *$ & $22.9 \pm 0.9$ & 1 & & \\
\hline & HB2017022 & 2017 & D & 311 & $* *$ & $20.2 \pm 1.3$ & 2 & & \\
\hline & HB2017023 & 2017 & D & 219 & $* *$ & $18.0 \pm 3.4$ & 3 & & \\
\hline & HB2017024 & 2017 & $\mathrm{D}$ & 150 & $* *$ & $21.2 \pm 1.0$ & 1 & & \\
\hline & HB2017025 & 2017 & $\mathrm{D}$ & 312 & $*$ & $8.0 \pm 5.0$ & 3 & & \\
\hline & HB2017027 & 2017 & $\mathrm{D}$ & 314 & $* * *$ & $24.7 \pm 2.7$ & 4 & & \\
\hline & HB2017030 & 2017 & D & 317 & $* *$ & $20.2 \pm 3.0$ & 1 & & \\
\hline & HB2017032 & 2017 & D & 319 & $* *$ & $19.7 \pm 1.3$ & 5 & & \\
\hline & HB2017034 & 2017 & E & 100 & $* * *$ & $19.4 \pm 2.5$ & 1 & & \\
\hline & HB2017035 & 2017 & E & 321 & $* *$ & $21.8 \pm 5.7$ & 1 & & \\
\hline
\end{tabular}


Table 1. Cont.

\begin{tabular}{|c|c|c|c|c|c|c|c|c|c|}
\hline $\begin{array}{c}\text { Clusters and } \\
\text { Species }\end{array}$ & Isolates & Year & Source & STs & Adhesion & LDH & NDR & ESBLs & $q n r$ \\
\hline & HB2017037 & 2017 & E & 323 & $* *$ & $24.8 \pm 6.8$ & 1 & & \\
\hline & HB2017041 & 2017 & F & 327 & * & $5.4 \pm 1.6$ & 1 & & \\
\hline & HB2017043 & 2017 & $\mathrm{~F}$ & 329 & * & $20.0 \pm 0.6$ & 1 & & \\
\hline & HB2017046 & 2017 & $\mathrm{~F}$ & 332 & ** & $22.9 \pm 7.0$ & 2 & & \\
\hline & HB2017047 & 2017 & F & 333 & ** & $23.5 \pm 5.0$ & 6 & & \\
\hline & HB2017049 & 2017 & F & 335 & * & $31.2 \pm 10.2$ & 2 & & \\
\hline & HB2017051 & 2017 & F & 214 & $* *$ & $21.0 \pm 4.4$ & 2 & & \\
\hline & HB2017055 & 2017 & $\mathrm{~F}$ & 161 & ** & $18.2 \pm 3.1$ & 6 & & \\
\hline & HB2017056 & 2017 & F & 340 & $* *$ & $19.2 \pm 3.4$ & 4 & & \\
\hline & HB2017057 & 2017 & F & 341 & $* *$ & $20.3 \pm 3.3$ & 4 & & \\
\hline \multirow{45}{*}{$\begin{array}{l}\text { Lineage III } \\
\text { C. braakii }\end{array}$} & HB2016015 & 2016 & $\mathrm{~F}$ & 280 & $+/-$ & $3.4 \pm 0.7$ & 5 & & \multirow{21}{*}{$a a c\left(6^{\prime}\right)-I b-c r, q n r B 2$} \\
\hline & HB2016032 & 2016 & E & 295 & $* *$ & $4.2 \pm 4.1$ & 5 & & \\
\hline & HB2016033 & 2016 & F & 296 & $* *$ & $5.3 \pm 3.2$ & 2 & & \\
\hline & HB2016035 & 2016 & $\mathrm{~F}$ & 297 & * & $11.1 \pm 2.2$ & 6 & & \\
\hline & HB2017044 & 2017 & F & 330 & $* *$ & $20.4 \pm 5.5$ & 2 & & \\
\hline & HB2017048 & 2017 & $\mathrm{~F}$ & 334 & * & $20.6 \pm 4.0$ & 2 & & \\
\hline & HB2017062 & 2017 & $\mathrm{~F}$ & 345 & * & $14.8 \pm 1.9$ & 2 & & \\
\hline & HB2017068 & 2017 & F & 351 & ** & $12.5 \pm 8.9$ & 2 & & \\
\hline & HB2017070 & 2017 & D & 353 & * & $9.5 \pm 0.4$ & 2 & & \\
\hline & HB2017071 & 2017 & D & 354 & * & $22.2 \pm 6.9$ & 2 & & \\
\hline & HB2017072 & 2017 & $\mathrm{D}$ & 355 & ** & $25.2 \pm 4.0$ & 1 & & \\
\hline & HB2017074 & 2017 & D & 356 & $* * *$ & $14.9 \pm 7.4$ & 2 & & \\
\hline & HB2017075 & 2017 & D & 357 & $* *$ & $25.2 \pm 4.0$ & 1 & & \\
\hline & HB2017076 & 2017 & $\mathrm{D}$ & 358 & $* *$ & $28.9 \pm 1.6$ & 4 & & \\
\hline & HB2017077 & 2017 & D & 357 & $* *$ & $13.6 \pm 0.2$ & 1 & & \\
\hline & HB2017078 & 2017 & D & 359 & $+/-$ & $13.7 \pm 5.7$ & 1 & & \\
\hline & HB2017079 & 2017 & E & 360 & * & $15.5 \pm 2.0$ & 3 & & \\
\hline & HB2017081 & 2017 & E & 362 & $* *$ & $17.7 \pm 0.8$ & 2 & & \\
\hline & HB2017082 & 2017 & $\mathrm{~F}$ & 363 & ** & $13.1 \pm 1.9$ & 1 & & \\
\hline & HB2017083 & 2017 & $\mathrm{~F}$ & 364 & $* *$ & $10.8 \pm 0.6$ & 1 & & \\
\hline & HB2017084 & 2017 & F & 365 & $* *$ & $13.9 \pm 2.0$ & 3 & & \\
\hline & HB2017087 & 2017 & $\mathrm{~F}$ & 367 & * & $3.5 \pm 4.0$ & 8 & & \multirow{7}{*}{$\begin{array}{c}a a c\left(6^{\prime}\right)-I b-c r \\
q n r B 2\end{array}$} \\
\hline & HB2017089 & 2017 & $\mathrm{~F}$ & 369 & $+/-$ & $11.7 \pm 9.7$ & 2 & & \\
\hline & HB2017090 & 2017 & F & 370 & $+/-$ & $4.5 \pm 1.7$ & 8 & & \\
\hline & HB2017091 & 2017 & F & 371 & - & $4.7 \pm 0.1$ & 3 & & \\
\hline & HB2017092 & 2017 & F & 372 & * & $11.7 \pm 10.7$ & 4 & & \\
\hline & HB2017093 & 2017 & $\mathrm{~F}$ & 373 & $+/-$ & $6.5 \pm 2.0$ & 2 & & \\
\hline & HB2017094 & 2017 & F & 81 & $+/-$ & $9.8 \pm 5.6$ & 3 & & \\
\hline & HB2017095 & 2017 & F & 225 & $* *$ & $6.2 \pm 2.5$ & 6 & $b l a_{\text {СТХ-М-9 }}$ & \multirow[t]{4}{*}{$q n r B 2$} \\
\hline & HB2017096 & 2017 & $\mathrm{~F}$ & 374 & $* *$ & $31.7 \pm 4.8$ & 6 & $b l a_{\mathrm{CTX}} \mathrm{M}-3$ & \\
\hline & HB2017097 & 2017 & F & 375 & $* *$ & $17.5 \pm 1.9$ & 0 & & \\
\hline & HB2017098 & 2017 & $\mathrm{~F}$ & 376 & $+/-$ & $14.2 \pm 6.5$ & 1 & & \\
\hline & HB2017099 & 2017 & E & 297 & $+/-$ & $17.1 \pm 1.1$ & 6 & & \multirow[t]{6}{*}{$a a c\left(6^{\prime}\right)-I b-c r$} \\
\hline & HB2017100 & 2017 & $\mathrm{~F}$ & 377 & $*$ & $17.9 \pm 2.2$ & 2 & & \\
\hline & HB2017101 & 2017 & $\mathrm{~F}$ & 378 & $* * *$ & $21.1 \pm 5.1$ & 2 & & \\
\hline & HB2017102 & 2017 & F & 379 & * & $25.0 \pm 4.2$ & 3 & & \\
\hline & HB2017103 & 2017 & $\mathrm{~F}$ & 380 & * & $0.7 \pm 0.4$ & 4 & & \\
\hline & HB2017104 & 2017 & F & 381 & $+/-$ & $14.0 \pm 1.7$ & 3 & & \\
\hline & HB2017105 & 2017 & $\mathrm{~F}$ & 382 & $* *$ & $13.9 \pm 2.9$ & 9 & $b l a_{\text {TEM-1 }}$ & \multirow[t]{3}{*}{$a a c\left(6^{\prime}\right)-I b-c r$} \\
\hline & HB2017106 & 2017 & $\mathrm{~F}$ & 383 & $* *$ & $18.6 \pm 0.3$ & 4 & & \\
\hline & HB2017107 & 2017 & F & 384 & * & $18.5 \pm 1.1$ & 7 & & \\
\hline & HB2017108 & 2017 & F & 385 & $* *$ & $13.2 \pm 3.1$ & 6 & $b l a_{\mathrm{CTX}-\mathrm{M}-9}$ & \multirow{4}{*}{$\begin{array}{c}a a c\left(6^{\prime}\right)-I b-c r \\
q n r B 2\end{array}$} \\
\hline & HB2017109 & 2017 & $\mathrm{~F}$ & 386 & * & $20.4 \pm 2.4$ & 1 & & \\
\hline & HB2017110 & 2017 & $\mathrm{~F}$ & 375 & $* *$ & $18.0 \pm 3.0$ & 0 & & \\
\hline & HB2017111 & 2017 & $\mathrm{~F}$ & 387 & $* *$ & $18.0 \pm 0.8$ & 2 & & \\
\hline Lineage IV & HB2016029 & 2016 & $\mathrm{D}$ & 292 & $+/-$ & $6.5 \pm 4.8$ & 1 & & \multirow{6}{*}{$a a c\left(6^{\prime}\right)-I b-c r$} \\
\hline \multirow{5}{*}{ C. youngae } & HB2017007 & 2017 & D & 237 & * & $8.4 \pm 2.3$ & 8 & & \\
\hline & HB2017021 & 2017 & D & 74 & * & $10.0 \pm 0.5$ & 3 & & \\
\hline & HB2017029 & 2017 & $\mathrm{D}$ & 316 & $* *$ & $16.5 \pm 2.5$ & 2 & & \\
\hline & HB2017067 & 2017 & $\mathrm{D}$ & 350 & $* *$ & $15.3 \pm 2.7$ & 1 & & \\
\hline & HB2017086 & 2017 & F & 258 & ** & $7.0 \pm 5.1$ & 2 & & \\
\hline
\end{tabular}


Table 1. Cont

\begin{tabular}{|c|c|c|c|c|c|c|c|c|c|}
\hline $\begin{array}{l}\text { Clusters and } \\
\text { Species }\end{array}$ & Isolates & Year & Source & STs & Adhesion & LDH & NDR & ESBLs & $q n r$ \\
\hline Lineage $\mathrm{V}$ & HB2016026 & 2016 & $\mathrm{D}$ & 183 & $* *$ & $17.2 \pm 0.9$ & 1 & & \\
\hline \multirow{9}{*}{ C. youngae } & HB2016027 & 2016 & D & 291 & ** & $28.3 \pm 0.5$ & 1 & & \\
\hline & HB2016028 & 2016 & $\mathrm{D}$ & 187 & $* * *$ & $27.4 \pm 1.2$ & 0 & & \\
\hline & HB2016031 & 2016 & $\mathrm{~F}$ & 294 & $* *$ & $18.7 \pm 2.7$ & 2 & & \\
\hline & HB2017063 & 2017 & D & 346 & * & $18.7 \pm 1.5$ & 2 & & \\
\hline & HB2017064 & 2017 & $\mathrm{D}$ & 347 & $* * *$ & $25.2 \pm 1.4$ & 0 & & \\
\hline & HB2017065 & 2017 & D & 348 & $* * *$ & $20.4 \pm 4.9$ & 7 & & \\
\hline & HB2017066 & 2017 & $\mathrm{D}$ & 349 & - & $19.5 \pm 3.2$ & 0 & & \\
\hline & HB2017069 & 2017 & $\mathrm{~F}$ & 352 & $+/-$ & $16.2 \pm 14.6$ & 2 & & \\
\hline & HB2017085 & 2017 & $\mathrm{~F}$ & 366 & $* *$ & $22.1 \pm 6.5$ & 1 & & \\
\hline
\end{tabular}

Adhesion index: ${ }^{* * *},>50 ; * *,>1$ and $<50 ; *,<1 ;+/-$ ambivalent or no adhesion; - , no adhesion. LDH (\% \pm SD): The lactate dehydrogenase released from Hep-2 cells; STs, sequence types; NDR, number of drugs resistance; D, F and E, isolates from diarrheal patients, foods and environment. ESBLs: extended-spectrum $\beta$-lactamase.

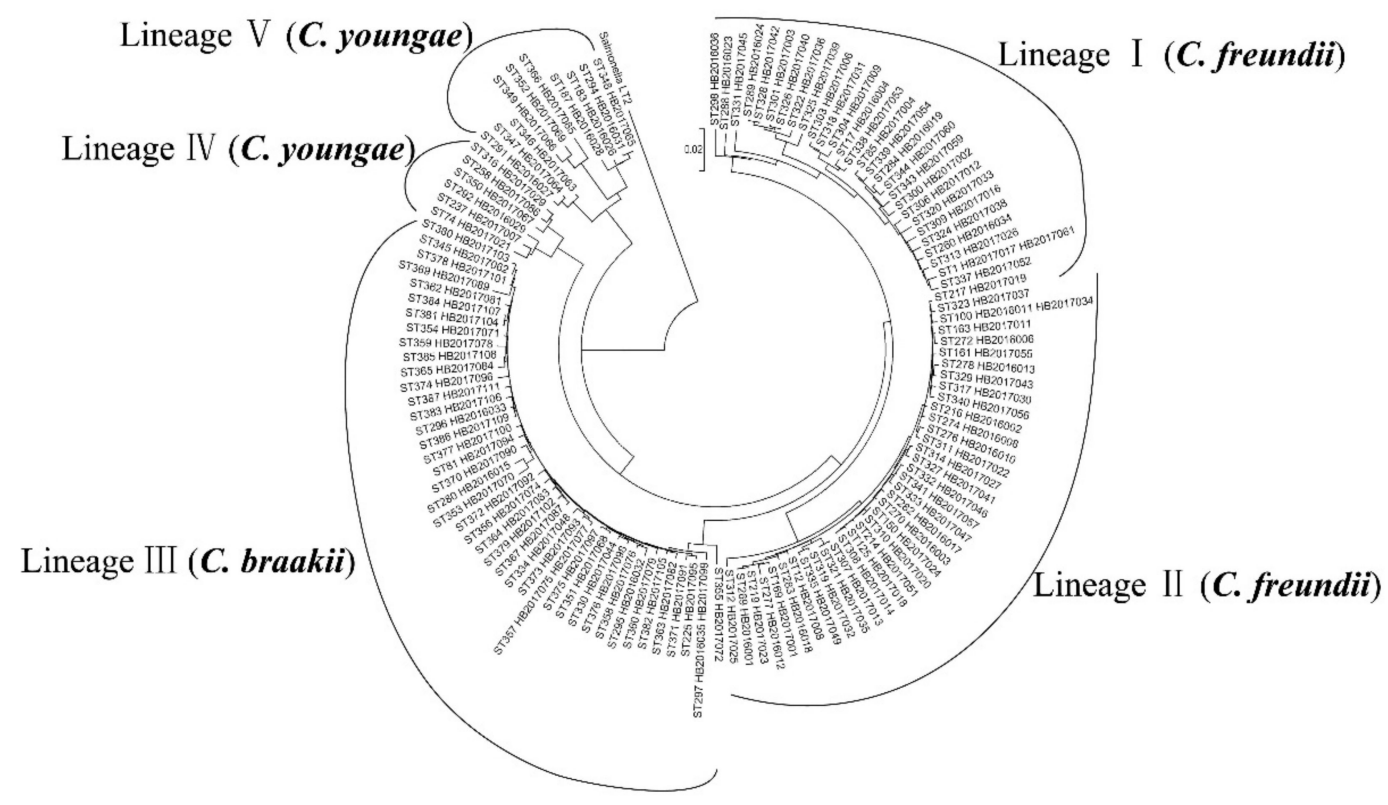

Figure 1. Phylogenetic relationships of the 128 Citrobacter isolates from this study. The phylogenetic tree of the 128 Citrobacter isolates was constructed using the concatenated sequences of the seven housekeeping genes by the neighbor-joining algorithm. Salmonella LT2 was used as an outgroup. Lineage divisions were marked. Bootstrap values of 50\% or more from 1000 replicates were shown.

The STs of C. freundii in Lineages I and II from this study were compared with 85 STs of $C$. freundii from Maanshan Anhui Province in our previous study [10,16], only nine STs found in this study shared the same STs from Maanshan city. C. freundii from these two regions displayed high diversity. All STs of $C$. freundii from this study and our previous study were used to construct a phylogenetic tree. The tree was divided into six clusters with strong bootstrap support of the cluster divisions (Figure 2 and Supplementary Figure S1). Cluster 1 to 5 were the same as previously defined [16]. However, a group within cluster 1 of our previous study is now a separate cluster and named cluster 6 . The STs of C. freundii in Lineage I and Lineage II from this study were equivalent to cluster 1 and cluster 2 in our previous study (Figure 2 and Supplementary Figure S1). 


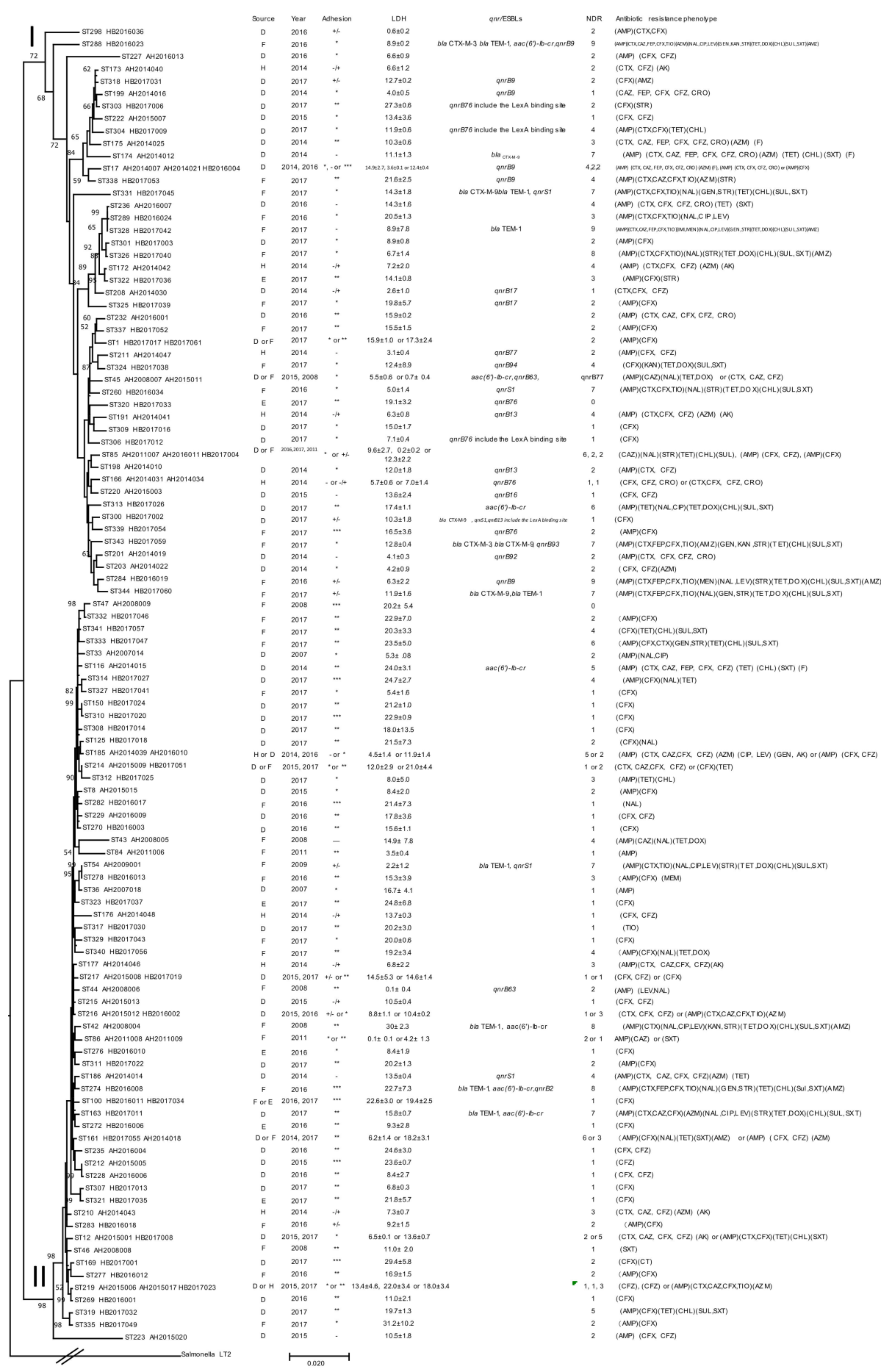

Figure 2. Phylogenetic relationships of the 123 C. freundii isolates from this study and two previous studies $[10,16]$. Lineages are marked on the node with roman numerals. Bootstrap values from 1000 replicates are shown on or near the nodes if $\geq 50 \%$. The presence of ESBL and $q n r$ genes, source, year, NDR (number of drugs resistant to), adhesion, LDH and antibiotic resistance phenotype of an isolate is shown on the right. The tree was constructed using the neighbor joining method. ST, D, F, E, H, and LDH denote sequence types, isolates from diarrheal patients, foods, environment and healthy individuals, and lactate dehydrogenase, respectively. Adhesion index: ${ }^{* *},>50 ;{ }^{* *},>1$ and $<50 ;{ }^{*},<1$; +/-, ambivalent or no adhesion; -, no adhesion. 
The 16 STs of $C$. youngae in Lineage IV and Lineage V from this study were compared with 32 STs of $C$. youngae from Maanshan, Anhui Province in our previous study. Fifteen STs from this study were novel STs, and there was little overlap of STs. All STs of $C$. youngae from this study and our previous study were used to construct a phylogenetic tree, the tree was divided into two clusters (cluster 1 and cluster 2) with strong bootstrap support of the cluster divisions, and 16 STs of $C$. youngae in Lineage IV and Lineage $\mathrm{V}$ from this study were equivalent to cluster 1 and cluster 2 in our previous study [10] (Figure 3),

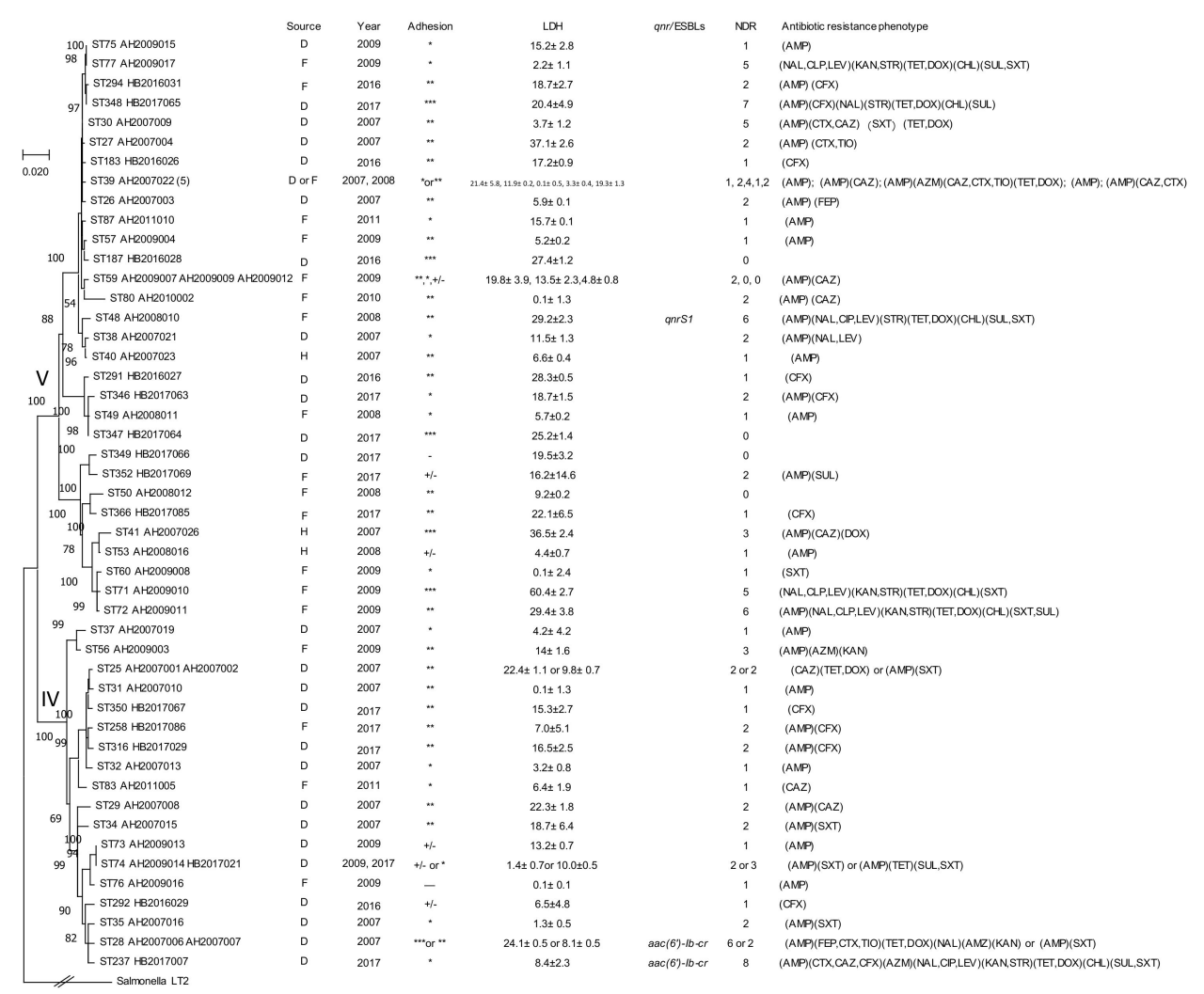

Figure 3. Phylogenetic relationships of the 57 C. youngae isolates from this study and our previous study [10]. Lineages divisions are marked on the node with roman numerals. The number in bracket after strain name denote number of strains for ST39 which includes AH2007022, AH2007024, AH2007025, AH2008001, AH2008002). Bootstrap values (numbers on or near the nodes) from 1000 replicates are shown if $\geq 50 \%$. The presence of ESBLs and qnr genes, source, year, NDR (the number of drugs resistant to), adhesion, $\mathrm{LDH}$ and antibiotic resistance phenotype of an isolate is shown on the right. The tree was constructed using the neighbor joining method. ST, D, F, H, and LDH denote sequence types, isolates from diarrheal patients, foods and healthy individuals, and lactate dehydrogenase, respectively. Adhesion index: ${ }^{* * *},>50 ;{ }^{* *},>1$ and $<50 ;{ }^{*},<1 ;+/-$, ambivalent or no adhesion; -, no adhesion.

The 42 STs of $C$. braakii in Lineage III from this study were compared with 8 STs of $C$. braakii from Maanshan Anhui Province in our previous study [10], and only one ST was common between the two regions. All STs of C. braakii from this study and our previous study were used to construct a phylogenetic tree (Figure 4), and all isolates belonged to the same cluster.

We further analyzed the 123 STs using eBURST $[24,25]$ to identify clonal complexes. In this study we defined CCs as STs shared six of the seven alleles to identify the most closely related STs [24,25]. We also retrieved all C. freundii STs from other countries from the public MLST database to identify CCs that include isolates from other countries. It should be noted that there is no C. youngae or $C$. braakii isolates from other countries in the MLST database. There were 27, 7 and 5 CCs identified for $C$. freundii, C. youngae and C. braakii isolates, respectively (Supplementary Table S1). For the 27 C. freundii 
CCs, 17 CCs included isolates from other countries with one CC containing isolates from five different countries, $10 \mathrm{CCs}$ included isolates from two different regions of China but no isolates from other countries, and only four CCs were restricted in the same region of China. For the seven C. youngae CCs, only one was from two different regions of China. For the five C. braakii CCs only one was from two different regions of China also.

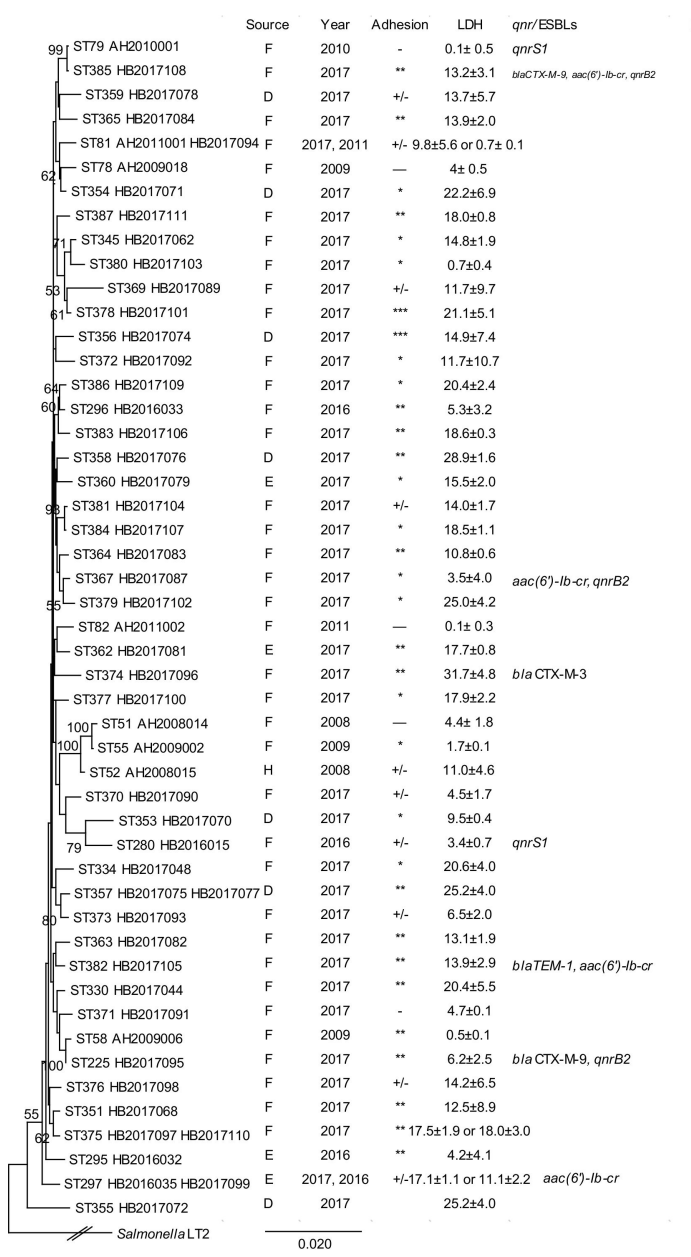

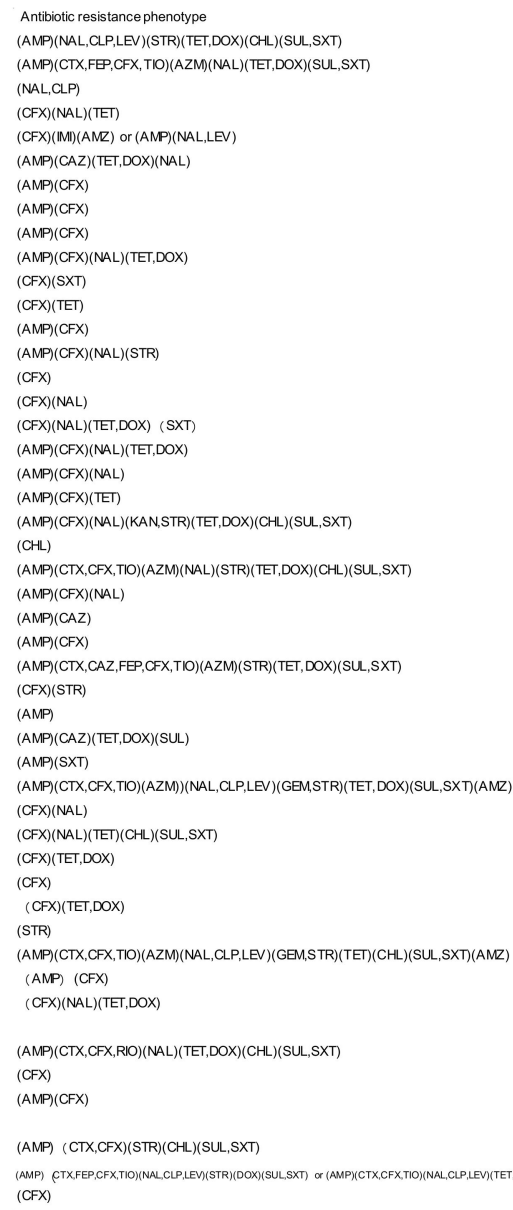

Figure 4. Phylogenetic relationships of the 53 C. braakii isolates (lineage III) from this study and our previous study [10]. The phylogenetic tree of the 53 C. braakii isolates was constructed using the concatenated sequences of the seven housekeeping genes by the neighbor-joining method. Bootstrap values of $50 \%$ or more from 1000 replicates were shown. The presence of ESBLs and qnr genes, source, year, NDR (number of drugs resistant to), adhesion, LDH and antibiotic resistance phenotype of an isolate is shown on the right. The tree was constructed using the neighbor joining algorithm. ST, D, F, $\mathrm{H}$, and LDH indicate sequence types, isolates from diarrheal patients, foods and healthy individuals, and lactate dehydrogenase, respectively. Adhesion index: ${ }^{* *},>50 ;{ }^{* *},>1$ and $<50 ; *,<1 ;+/-$, ambivalent or no adhesion; -, no adhesion.

\subsection{Prevalence of Antimicrobial Resistance}

Susceptibility to 22 antibiotics was tested on the 128 Citrobacter isolates using the broth microdilution method according to CLSI recommendations (Table 2). All isolates were sensitive to amikacin (AMI). For the $67 \mathrm{C}$. freundii isolates, most were resistant to one or more of the $\beta$-lactams, especially to penicillins (58.2\%), cephalosporins (9.0-94.0\%), monobactams (7.5\%) and carbapenems (1.5-4.5\%). Resistance to the three quinolones tested ranged from $7.5 \%$ to $23.9 \%$. Resistance to other antibiotics was as follows: Aminoglycosides (0-20.9\%), tetracyclines (16.4-32.8\%), phenicols $(25.4 \%)$, sulfonamides (22.4-25.4\%) and macrolides (10.4\%). For the 45 C. braakii isolates, resistance to different 
$\beta$-lactams was as follows: Penicillins (51.1\%), cephalosporins (3.0-88.9\%), monobactams $(11.1 \%)$ and carbapenems $(0-2.2 \%)$, and resistance to quinolones $(6.7-44.4 \%)$, aminoglycosides $(0-22.2 \%)$, tetracyclines (31.1-42.2\%), phenicols (20.0\%), sulfonamides $(24.4-28.9 \%)$ and macrolides $(6.7 \%)$. For 16 C. youngae isolates, varied resistance was found to penicillins ( $50.0 \%)$, cephalosporins (0-68.8\%), monobactams $(6.3 \%)$, quinolones $(6.3-12.5 \%)$, aminoglycosides $(0-12.5 \%)$, tetracyclines $(12.5-18.8 \%)$, phenicols (12.5\%) and sulfonamides (18.8\%).

Resistance to at least one antibiotic of three or more distinct classes was defined as multidrug-resistant (MDR). We found 51 MDR isolates which were distributed in five lineages, and mainly in Lineages I, II, and III which included 14/29 (48.3\%), 13/38 (34.2\%) and 21/45 (46.7\%) MDR isolates, respectively. Ten of the 51 MDR isolates were isolated from 2016, and the remaining 41 were from 2017; By source, 13 of the 51 MDR isolates (25.5\%) were isolated from diarrheal patient, four $(7.8 \%)$ from the environment and $34(66.7 \%)$ from foods (Table 1$)$.

There were 27 MDR C. freundii isolates, including 9/30 (30.0\%) from diarrheal patient, 17/30 (56.7\%) from foods and 1/7 (14.3\%) from the environment (Table 2). A total of 58 MDR C. freundii isolates were isolated from this study and our previous study, including 28/94 (29.8\%) from diarrheal patient, $22 / 41(63.7 \%)$ from foods, $7 / 19(36.8 \%)$ from healthy individuals and 1/7 (14.3\%) from the environment (Supplementary Table S2).

For C. youngae, 3 MDR isolates were isolated from this study, including 3/12 (25.0\%) from diarrheal patient (Table 2) and together with 9 MDR isolates from our previous study, 5/30 (16.7\%) were from diarrheal patient, $6 / 24(25.0 \%)$ from foods and $1 / 3(33.3 \%)$ from healthy individuals (Supplementary Table S2).

For C. braakii, 21 MDR isolates were isolated from this study, including 1/8 (12.5\%) from diarrheal patient, $17 / 33(51.5 \%)$ from foods and $3 / 4$ (75.0\%) from the environment (Table 2). Five MDR isolates were isolated from our previous study. Taken together, the MDR rate was 1/8 (12.5\%) from diarrheal patient, 22/40 (55.0\%) from foods and 3/4 (75.0\%) from the environment (Supplementary Table S2).

\subsection{Prevalence of ESBLs and Fluoroquinolone Resistance}

Among the 128 Citrobacter isolates, 12 ESBL isolates were detected, which harbored $b l a_{\mathrm{CTX}-\mathrm{M}-3}$, $b l a_{\mathrm{CTX}-\mathrm{M}-9}$ or $b l a_{\mathrm{TEM}-1}, 11$ of which were MDR. No isolate carried $b l a_{S H V}, b l a_{\text {GES }}, b l a_{P E R}$ or $b l a_{V E B}$ genes.

Thirty eight $(29.7 \%)$ of the 128 Citrobacter isolates were resistant to fluoroquinolones, including 16 C. freundii, 2 C. youngae and 20 C. braakii isolates, all of which were resistant to NAL (MICs $\geq 32$ $\mu \mathrm{g} / \mathrm{mL}$ ); 12 resistant to CIP (MICs $\geq 2 \mu \mathrm{g} / \mathrm{mL}$ ); and 10 resistant to LEV (MICs $\geq 8 \mu \mathrm{g} / \mathrm{mL}$ ). By source, 27 $(71.1 \%)$ of the 38 fluoroquinolones resistant isolates were from food, two from the environment and nine from diarrheal patients. These isolates were distributed among different phylogenetic lineages, 9/38 (23.7\%) in Lineage I, 7/38 (1.8\%) in Lineage II, 20/38 (52.6\%) in Lineage III, one each in Lineage IV and V. Thirty three $(86.8 \%$ ) of the 38 fluoroquinolones resistant isolates were MDR (MDR $\geq 3$ ), and 9 $(23.7 \%)$ were EMBLs carrying the $b l a_{\mathrm{CTX}-\mathrm{M}-3}, b l a_{\mathrm{CTX}-\mathrm{M}-9}$, or $b l a_{\mathrm{TEM}-1}$ gene (Table 3$)$.

Twenty eight (73.7\%) of the 38 NAL-resistant isolates analyzed contained mutations in the QRDR of the gyrA gene, with 27 having one mutation in codon 59 (Thr59Ile) and one having three mutations in codons 59, 111and 134 (Thr59Ile, Gln111Arg and Ile134Val). No mutations were found in the QRDR region of the parC gene (Table 3).

Of the 28 NAL-resistant isolates with gyrA mutations, 12 belonged to $C$. freundii, 14 to $C$. braakii, 2 to $C$. youngae. Among the $12 C$. freundii isolates with gyrA mutations, six were resistant to CIP (MIC of $\geq 4 \mu \mathrm{g} / \mathrm{mL}$ ), and five were resistant to LEV (MIC of $\geq 8 \mu \mathrm{g} / \mathrm{mL}$ ); of the 14 C. braakii with gyrA mutations, three were resistant to CIP (MIC of $8 \mu \mathrm{g} / \mathrm{mL}$ ), and three to LEV (MIC of $\geq 8 \mu \mathrm{g} / \mathrm{mL}$ ); of the two C. youngae isolates with gyrA mutations, only one was resistant to CIP and LEV (MIC of $8 \mu \mathrm{g} / \mathrm{mL}$ ) (Table 3). 
Table 2. Prevalence of resistance to different antibiotics by species and source.

\begin{tabular}{|c|c|c|c|c|c|c|c|c|c|}
\hline \multirow[t]{2}{*}{ Antibiotic } & \multicolumn{3}{|c|}{ C. freundii $(\mathrm{n}=67)$ Resistance $(\%)$} & \multicolumn{3}{|c|}{ C. youngae $(\mathrm{n}=16)$ Resistance $(\%)$} & \multicolumn{3}{|c|}{ C.braakii $(\mathrm{n}=45)$ Resistance $(\%)$} \\
\hline & $D(n=30)$ & $F(n=30)$ & $E(n=7)$ & $D(n=12)$ & $F(n=4)$ & $E(n=0)$ & $D(n=8)$ & $F(n=33)$ & $E(n=4)$ \\
\hline \multicolumn{10}{|l|}{ PENICILLINS } \\
\hline Ampicillin & $15(50.0)$ & $23(76.7)$ & $1(14.3)$ & $5(41.7)$ & $3(75.0)$ & $0(0)$ & $3(37.5)$ & $16(48.5)$ & $4(100.0)$ \\
\hline \multicolumn{10}{|l|}{ CEPHALOSPORINS } \\
\hline Cefotaxime & $6(20.0)$ & $12(40.0)$ & $0(0)$ & $1(8.3)$ & $0(0)$ & $0(0)$ & $0(0)$ & $7(21.2)$ & $2(50.0)$ \\
\hline Ceftazidime & $3(10.0)$ & $3(10.0)$ & $0(0)$ & $1(8.3)$ & $0(0)$ & $0(0)$ & $0(0)$ & $1(3.0)$ & $0(0)$ \\
\hline Cefepime & $0(0)$ & $6(20.0)$ & $0(0)$ & $0(0)$ & $0(0)$ & $0(0)$ & $0(0)$ & $1(3.0)$ & $0(0)$ \\
\hline Cefoxitin & $28(93.3)$ & $29(96.7)$ & $6(85.7)$ & $8(66.7)$ & $3(75.0)$ & $0(0)$ & $7(87.5)$ & $29(87.9)$ & $4(100.0)$ \\
\hline Ceftiofur Sodium & $3(10.0)$ & $11(36.7)$ & $0(0)$ & $0(0)$ & $0(0)$ & $0(0)$ & $0(0)$ & $7(21.2)$ & $1(25.0)$ \\
\hline \multicolumn{10}{|l|}{ MONOBACTAMS } \\
\hline Aztreonam & $2(6.7)$ & $3(10.0)$ & $0(0)$ & $1(8.3)$ & $0(0)$ & $0(0)$ & $0(0)$ & $5(15.2)$ & $0(0)$ \\
\hline \multicolumn{10}{|l|}{ CARBAPENEMS } \\
\hline Imipenem & $0(0)$ & $1(3.3)$ & $0(0)$ & $0(0)$ & $0(0)$ & $0(0)$ & $0(0)$ & $1(3.0)$ & $0(0)$ \\
\hline Meropenem & $0(0)$ & $3(10.0)$ & $0(0)$ & $0(0)$ & $0(0)$ & $0(0)$ & $0(0)$ & $0(0)$ & $0(0)$ \\
\hline \multicolumn{10}{|l|}{ QUINOLONES } \\
\hline Nalidixicacid & $4(13.3)$ & $12(40.0)$ & $0(0)$ & $2(16.7)$ & $0(0)$ & $0(0)$ & $3(37.5)$ & $15(45.5)$ & $2(50.0)$ \\
\hline Ciprofloxacin & $2(6.7)$ & $4(13.3)$ & $0(0)$ & $1(8.3)$ & $0(0)$ & $0(0)$ & $0(0)$ & $3(9.1)$ & $0(0)$ \\
\hline Levofloxacin & $1(3.3)$ & $4(13.3)$ & $0(0)$ & $1(8.3)$ & $0(0)$ & $0(0)$ & $0(0)$ & $3(9.1)$ & $0(0)$ \\
\hline \multicolumn{10}{|l|}{ AMINOGLYCOSIDES } \\
\hline Gentamicin & $0(0)$ & $7(23.3)$ & $0(0)$ & $0(0)$ & $0(0)$ & $0(0)$ & $0(0)$ & $2(6.1)$ & $0(0)$ \\
\hline Amikacin & $0(0)$ & $0(0)$ & $0(0)$ & $0(0)$ & $0(0)$ & $0(0)$ & $0(0)$ & $0(0)$ & $0(0)$ \\
\hline Streptomycin & $2(6.7)$ & $11(36.7)$ & $1(14.3)$ & $2(16.7)$ & $0(0)$ & $0(0)$ & $0(0)$ & $8(24.2)$ & $2(50.0)$ \\
\hline Kanamycin & $0(0)$ & $3(10.0)$ & $0(0)$ & $1(8.3)$ & $0(0)$ & $0(0)$ & $0(0)$ & $1(3.0)$ & $0(0)$ \\
\hline \multicolumn{10}{|l|}{ TETRACYCLINES } \\
\hline Tetracycline & $7(23.3)$ & $15(50.0)$ & $0(0)$ & $3(25.0)$ & $0(0)$ & $0(0)$ & $1(12.5)$ & $17(51.5)$ & $1(25.0)$ \\
\hline Doxycycline & $2(6.7)$ & $9(30.0)$ & $0(0)$ & $2(16.7)$ & $0(0)$ & $0(0)$ & $1(12.5)$ & $12(36.4)$ & $1(25.0)$ \\
\hline \multicolumn{10}{|l|}{ PHENICOLS } \\
\hline Chloramphenicol & $6(20.0)$ & $11(36.7)$ & $0(0)$ & $2(16.7)$ & $0(0)$ & $0(0)$ & $0(0)$ & $7(21.2)$ & $2(50.0)$ \\
\hline \multicolumn{10}{|l|}{ SULFONAMIDES } \\
\hline Trimethoprim/Sulfamethoxazole & $4(13.3)$ & $13(43.3)$ & $0(0)$ & $2(16.7)$ & $1(25.0)$ & $0(0)$ & $0(0)$ & $11(33.3)$ & $2(50.0)$ \\
\hline Sulfafurazole & $3(10.0)$ & $12(40.0)$ & $0(0)$ & $3(25.0)$ & $0(0)$ & $0(0)$ & $0(0)$ & $9(37.3)$ & $2(50.0)$ \\
\hline \multicolumn{10}{|l|}{ MACROLIDES } \\
\hline Azithromycin & $1(3.3)$ & $6(20.0)$ & $0(0)$ & $0(0)$ & $0(0)$ & $0(0)$ & $0(0)$ & $3(9.1)$ & $0(0)$ \\
\hline MDR & $9(30.0)$ & $17(56.7)$ & $1(14.3)$ & $3(25.0)$ & $0(0)$ & $0(0)$ & $1(12.5)$ & $17(51.5)$ & $3(75.0)$ \\
\hline
\end{tabular}

D, diarrheal patients; F, foods; E, environment. MDR: With resistance to at least one antibiotic of three or more distinct classes (MDR $\geq 3$ ). 
Table 3. Quinolone resistant Citrobacter isolates and the presence of quinolone resistance genes and alterations in the gyr $A$ gene.

\begin{tabular}{|c|c|c|c|c|c|c|c|c|c|c|}
\hline Isolates & Species & Year & Source & ST & NDR & NAL & $\mathrm{CIP}$ & LEV & PMQR & gyrA Position \\
\hline HB2016008 & C. freundii & 2016 & $\mathrm{~F}$ & 274 & 8 & $>128$ & 4 & & $a a c\left(6^{\prime}\right)-I b-c r, q n r B 2$ & Thr59Ile \\
\hline HB2016017 & C. freundii & 2016 & $\mathrm{~F}$ & 282 & 1 & $>128$ & & & & Thr59Ile \\
\hline HB2017011 & C. freundii & 2017 & $\mathrm{D}$ & 163 & 7 & $>64$ & 16 & 8 & $a a c\left(6^{\prime}\right)-I b-c r$ & Thr59Ile \\
\hline HB2017018 & C. freundii & 2017 & $\mathrm{D}$ & 125 & 2 & $>128$ & & & & Thr59Ile \\
\hline HB2017027 & C. freundii & 2017 & $\mathrm{D}$ & 314 & 4 & $>64$ & & & & No mutation \\
\hline HB2017055 & C. freundii & 2017 & $\mathrm{~F}$ & 161 & 6 & $>128$ & & & & Thr59Ile \\
\hline HB2017056 & C. freundii & 2017 & $\mathrm{~F}$ & 340 & 4 & $>128$ & & & & Thr59Ile \\
\hline HB2016019 & C. freundii & 2016 & $\mathrm{~F}$ & 284 & 9 & $>128$ & & 8 & qnrB9 & Thr59Ile \\
\hline HB2016023 & C. freundii & 2016 & $\mathrm{~F}$ & 288 & 9 & $>128$ & 32 & 16 & $\begin{array}{c}a a c\left(6^{\prime}\right)-I b-c r \\
\text { gnrB9 }\end{array}$ & Thr59Ile \\
\hline HB2016024 & C. freundii & 2016 & $\mathrm{~F}$ & 289 & 3 & $>128$ & 4 & 8 & & Thr59Ile \\
\hline HB2016034 & C. freundii & 2016 & $\mathrm{~F}$ & 260 & 7 & 32 & & & qnrS1 & No mutation \\
\hline HB2017026 & C. freundii & 2017 & $\mathrm{D}$ & 313 & 6 & $>128$ & 4 & & $a a c\left(6^{\prime}\right)-I b-c r$ & Thr59Ile \\
\hline HB2017040 & C. freundii & 2017 & $\mathrm{~F}$ & 326 & 8 & $>128$ & & & & Thr59Ile \\
\hline HB2017042 & C. freundii & 2017 & $\mathrm{~F}$ & 328 & 9 & $>128$ & 8 & 16 & & Thr59Ile \\
\hline HB2017045 & C. freundii & 2017 & $\mathrm{~F}$ & 331 & 7 & 64 & & & $q n r S 1$ & No mutation \\
\hline HB2017060 & C. freundii & 2017 & $\mathrm{~F}$ & 344 & 7 & $>128$ & & & & No mutation \\
\hline HB2016015 & C. braakii & 2016 & $\mathrm{~F}$ & 280 & 5 & $>128$ & & & qnrS1 & Thr59Ile \\
\hline HB2016033 & C. braakii & 2016 & $\mathrm{~F}$ & 296 & 2 & $>128$ & & & & Thr59Ile \\
\hline HB2016035 & C. braakii & 2016 & $\mathrm{~F}$ & 297 & 6 & $>128$ & 8 & 8 & $\begin{array}{c}a a c\left(6^{\prime}\right)-I b-c r \\
q n r B 2\end{array}$ & Thr59Ile \\
\hline HB2017070 & C. braakii & 2017 & $\mathrm{D}$ & 353 & 2 & $>64$ & & & & Thr59Ile \\
\hline HB2017076 & C. braakii & 2017 & $\mathrm{D}$ & 358 & 4 & $>128$ & & & & Thr59Ile \\
\hline HB2017078 & C. braakii & 2017 & $\mathrm{D}$ & 359 & 1 & $>64$ & 2 & & & No mutation \\
\hline HB2017079 & C. braakii & 2017 & E & 360 & 3 & $>128$ & & & & Thr59Ile \\
\hline HB2017084 & C. braakii & 2017 & $\mathrm{~F}$ & 365 & 3 & $>64$ & & & & Thr59Ile \\
\hline HB2017087 & C. braakii & 2017 & F & 367 & 8 & $>128$ & & & $\begin{array}{c}a a c\left(6^{\prime}\right)-I b-c r \\
q n r B 2\end{array}$ & Thr59Ile \\
\hline HB2017090 & C. braakii & 2017 & $\mathrm{~F}$ & 370 & 8 & $>128$ & 8 & $>16$ & & Thr59Ile \\
\hline HB2017091 & C. braakii & 2017 & $\mathrm{~F}$ & 371 & 3 & $>128$ & & & & Thr59Ile \\
\hline HB2017092 & C. braakii & 2017 & $\mathrm{~F}$ & 372 & 4 & 64 & & & & No mutation \\
\hline HB2017095 & C. braakii & 2017 & $\mathrm{~F}$ & 225 & 6 & $>128$ & & & $q n r B 2$ & No mutation \\
\hline HB2017099 & C. braakii & 2017 & E & 297 & 6 & $>128$ & 8 & 8 & $a a c\left(6^{\prime}\right)-I b-c r$ & Thr59Ile \\
\hline HB2017102 & C. braakii & 2017 & $\mathrm{~F}$ & 379 & 3 & 32 & & & & No mutation \\
\hline HB2017103 & C. braakii & 2017 & $\mathrm{~F}$ & 380 & 4 & $>128$ & & & & Thr59Ile \\
\hline HB2017105 & C. braakii & 2017 & $\mathrm{~F}$ & 382 & 9 & $>128$ & 8 & 16 & $a a c\left(6^{\prime}\right)-I b-c r$ & No mutation \\
\hline HB2017106 & C. braakii & 2017 & $\mathrm{~F}$ & 383 & 4 & 32 & & & & No mutation \\
\hline HB2017107 & C. braakii & 2017 & $\mathrm{~F}$ & 384 & 7 & $>128$ & & & & Thr59Ile \\
\hline HB2017108 & C. braakii & 2017 & $\mathrm{~F}$ & 385 & 6 & $>128$ & & & $\begin{array}{c}a a c\left(6^{\prime}\right)-I b-c r \\
q n r B 2\end{array}$ & Thr59Ile \\
\hline HB2017007 & C. youngae & 2017 & $\mathrm{D}$ & 237 & 8 & $>64$ & 8 & 8 & $a a c\left(6^{\prime}\right)-I b-c r$ & Thr59Ile \\
\hline HB2017065 & C. youngae & 2017 & $\mathrm{D}$ & 348 & 7 & $>128$ & & & & Thr59Ile, Gln111Arg, Ile134Val \\
\hline
\end{tabular}

NAL, nalidixicacid; CIP, ciprofloxacin; LEV, levofloxacin. NDR, number of drugs resistant to. PMQR, plasmid-mediated quinolone resistance (PMQR) genes. 


\subsection{Prevalence of qnrB Genes}

Nineteen Citrobacter isolates, including 4 C. braakii and 15 C. freundii isolates, were found to harbor qnrB genes (including qnrB2, qnrB9, qnrB17, qnrB76, qnrB13, qnrB93 and qnrB94) (Table 1). Four C. braakii and one $C$. freundii isolates harbored $q n r B 2$, all of which were resistant to NAL, (MICs $\geq 128$ $\mu \mathrm{g} / \mathrm{mL}$ ), and were MDR. QnrB9 was found in five C. freundii isolates with three (HB2017053, HB2016023 and HB2017031) isolated from food and MDR, and two (HB2016004 and HB2017031) isolated from diarrheal patients and none was MDR. Among the three qnrB9-carrying MDR isolates, two (HB2016023 and HB2017031) were resistant to NAL (MICs $\geq 128 \mu \mathrm{g} / \mathrm{mL}$ ). QnrB17 was harbored in one C. freundii isolate (HB2017039) which was isolated from food and was not MDR. qnrB76 was harbored by two C. freundii isolates with one from food and one from the environment. A variant of $q n r B 76$ ( $q n r B 76$ contained a LexA binding site) was harbored by three $C$. freundii isolates, all of which were isolated from diarrheal patients. A variant of qnrB13 ( $q n r B 13$ contained a LexA binding site) was harbored by one C. freundii (HB2017002) isolate, which was isolated from a diarrheal patient.

Two isolates (HB2017059 and HB2017038) were found to harbor a new qnrB gene, both of which were isolated from food (Table 1). Sequence analysis revealed that the new qnrB gene harbored by HB2017059 differed from the qnrB13 gene (GenBank accession no. EU273756.1) by one nucleotide change (a $\mathrm{G} \rightarrow \mathrm{A}$ change at nt85 resulting in Asp $\rightarrow$ Asn), and this new qnrB allele was assigned qnrB93 (GenBank accession no.MK047606). The new qnrB gene harbored by HB2017038 differed from the qnrB11 gene (GenBank accession no. EU136183.1) by seven nucleotide change, including two non-synonymous changes, and $\mathrm{A} \rightarrow \mathrm{G}$ change at nt 430 resulted in $\mathrm{Thr} \rightarrow \mathrm{Ala}$ and an $\mathrm{A} \rightarrow \mathrm{C}$ change at nt556 resulted in Ile $\rightarrow$ Leu, and five synonymous changes, a $T \rightarrow G$ change at $n t 246, A \rightarrow C$ change at $\mathrm{nt} 357, \mathrm{G} \rightarrow \mathrm{A}$ change at nt399, $\mathrm{C} \rightarrow \mathrm{T}$ change at $\mathrm{nt} 468$ and $\mathrm{G} \rightarrow \mathrm{C}$ change at nt564. This new qnrB allele was assigned qnrB94 (GenBank accession no.MK047607) [26].

qnrB genes were predominantly harboured by Lineage I (C. freundii) (Table 1). When all C. freundii data combined, 26/53 (49.1\%) Lineage I isolates carried a qnrB allele (Figure 2).

\subsection{Adherence and Cytotoxicity of Citrobacter Isolates}

The 128 Citrobacter isolates were tested for adhesion and cytotoxicity to Hep-2 cells as done previously [10] (Table 1). Fourteen isolates (10.9\%) had an adhesion index greater than 50 and were classified as high adhesion. Fifty-seven (44.5\%) isolates had an adhesion index between 1 and 50 and were regarded as intermediate adhesive. Thirty seven (28.9\%) isolates showed little adhesion (adhesion index of $<1$ ). The remaining $20(15.6 \%)$ isolates showed ambivalent adhesion or no adhesion. By cytotoxicity, of the 128 isolates, $13(10.2 \%)$ were highly cytotoxic, 40 (31.3\%) intermediate cytotoxic and $75(58.6 \%)$ non-cytotoxic.

Among the 14 highly adhesive isolates, four isolates released LDH more than $24 \%$, and were considered highly cytotoxic (Figure 5); seven isolates released LDH from $19.4 \%$ to $22.9 \%$ and were considered intermediate cytotoxic; the remaining three isolates showed LDH release less than $16.5 \%$ and were likely to be non-cytotoxic (Table 1 and Supplementary Table S3).

Among the 57 intermediate adhesive isolates, seven isolates showed high cytotoxicity with more than $24 \%$ LDH released (Figure 5); 23 isolates released LDH from $18.0 \%$ to $23.5 \%$ and were intermediate cytotoxic, the remaining 27 isolates were considered to be non-cytotoxic (Table 1 and Supplementary Table S3). 


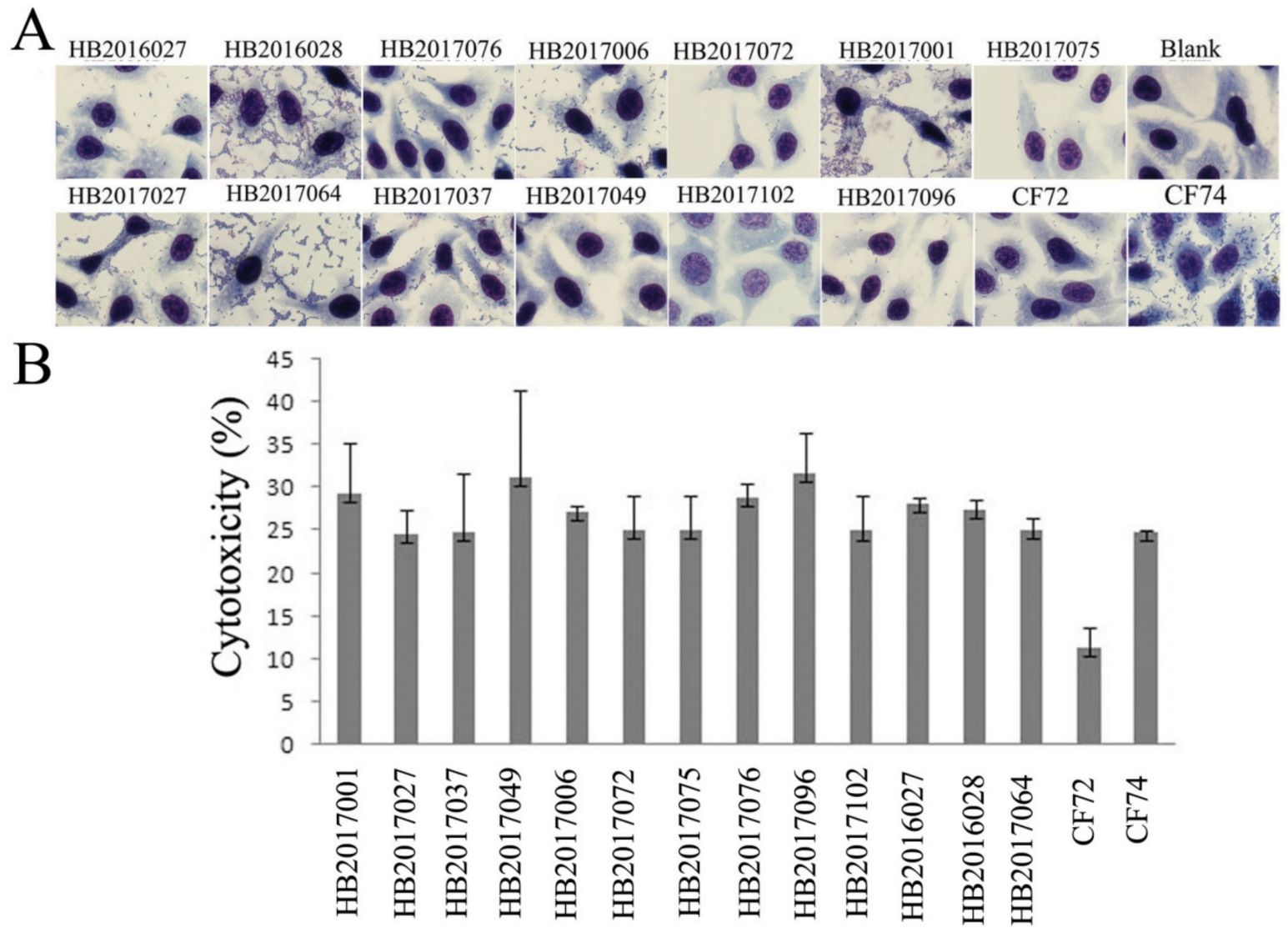

Figure 5. Adhesion and cytotoxicity of Citrobacter isolates to the human epidermoid laryngocarcinoma (Hep-2, CCC0068) cell line. (A) Light micrographs of the adherence patterns exhibited by the 13 highly cytotoxic Citrobacter isolates, and control strains CF74 and CF72. Bar: 10 $\mu \mathrm{m}$. (B) Cytotoxicity of the 13 highly cytotoxic Citrobacter isolates was measured by the amount of the LDH released after $8 \mathrm{~h}$ exposure by Hep-2 cells. CF72 and CF74 were control strains. CF72 was a non-cytotoxic and nonadhesive negative control, CF74 was a highly adherent and cytotoxic positive control.

Among the 37 less adhesive isolates, two showed high cytotoxicity with more than $24 \% \mathrm{LDH}$ released (Figure 5); nine were considered intermediate cytotoxic which released LDH from $17.9 \%$ to $22.2 \%$; the remaining 26 isolates showed LDH release less than $15.9 \%$ and are likely to be non-cytotoxic (Table 1 and Supplementary Table S3).

The 20 non adhesive isolates were also non-cytotoxic with all, except one showing intermediate cytotoxicity, releasing LDH from $0.6 \%$ to $17.1 \%$ (Table 1 and Supplementary Table S3).

We examined any differences in adhesion and cytotoxicity between lineages. We analyzed the difference using data in this study alone (Figure 6A,C) and also using combined data with our two previous studies (Figure 6B,D). Between Lineages I and II which exclusively contained C. freundii isolates, Lineage II showed higher proportion of high or intermediate adhesive and cytotoxic isolates than in Lineage I and the difference is statistically significant $(p<0.01)$ (Figure 6); Between Lineage IV and $\mathrm{V}$ which contained only $\mathrm{C}$. youngae isolates, the percentage of highly adhesive isolates in Lineage $\mathrm{V}$ was higher than in Lineage IV $(p<0.01)$ (Figure $6 \mathrm{~A}, \mathrm{C})$, and the percentage of the highly or intermediate cytotoxic isolates in Lineage $\mathrm{V}$ was also higher than in Lineage IV $(p<0.05)$ (Figure 6B,D). When the two virulence traits were considered together, Lineages II and V had higher adhesive and cytotoxic isolates than Lineages I, III, and IV (Figure 7). 

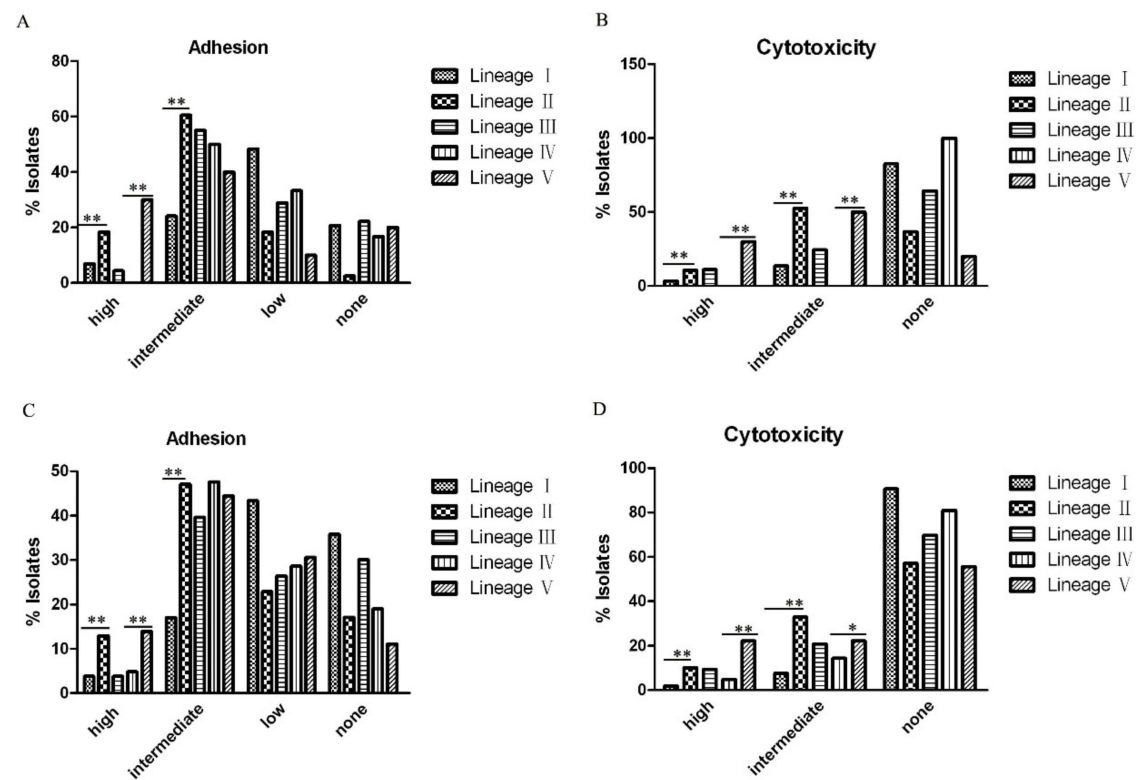

Figure 6. The percentage of adhesive and cytotoxic isolates in different lineages. (A) and (B) The percentage of high, intermediate, little or no adhesive or cytotoxic isolates based on the 128 Citrobacter isolates from this study. (C) and (D) The percentage of high, intermediate, little or no adhesive or cytotoxic isolates in different lineages based on the128 Citrobacter isolates from this study and 95 Citrobacter isolates from our previous studies [10,16]. The statistical significance between Lineages I and II or Lineages IV and V was determined by Mann-Whitney U test. ${ }^{*}, p<0.05 ;{ }^{* *}, p<0.01$.

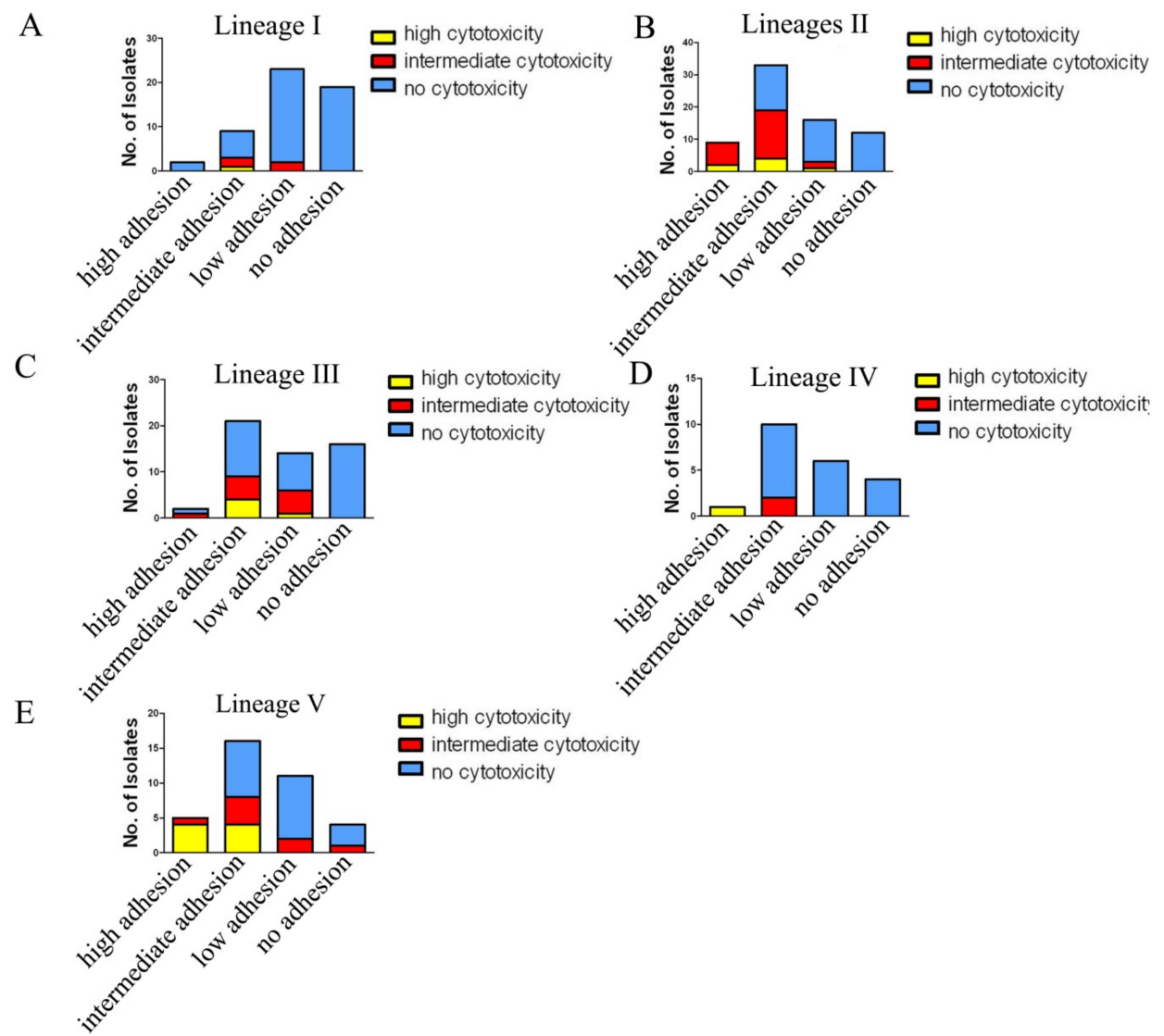

Figure 7. The number of adhesive and cytotoxic isolates in different lineages. The data were based on 128 isolates from this study and 95 Citrobacter isolates from our previous studies [10,16]. 


\section{Discussion}

Citrobacter spp. are opportunistic pathogens that can cause diarrhea, septicemia, meningitis, and urinary tract infections, especially in immunocompromised patients [27]. Together with our previous studies [10,16], we found 25 highly cytotoxic Citrobacter isolates out of $271(9.2 \%), 15$ were isolated from diarrheal patients ( $11.4 \%$ of diarrheal isolates), seven from foods ( $6.7 \%$ food isolates), two from healthy individuals (8.7\%) and 1 from the environment $(9.1 \%)$. The 22 highly cytotoxic Citrobacter isolates were distributed among the five lineages identified. However, Lineage II (C. freundii) and Lineage $\mathrm{V}$ (C. youngae) disproportionally contained more adhesive and more cytotoxic isolates than Lineages I, III, and IV and are likely to pose a higher risk to human health.

\subsection{High Genetic Diversity of Citrobacter spp. Across China and Internationally.}

The 128 Citrobacter isolates were divided into 123 STs, displaying high genetic diversity. We compared our STs with 268 STs from the Citrobacer MLST database and found 22 STs in this study were present in the database with isolates from other countries or regions, or from different sources. Among these 22 STs, ST1 contained isolates from diarrheal patients, healthy individuals, animals, insects and environment in our previous study [9]; ST12 contained isolates from a rectal swab (Israel in 2009) and a diarrheal patient (China in 2015) [16]; ST17 contained isolates from a skin necrosis, urine (Poland, 2012), two rectal swabs (Latvia, 2008 and 2009), and two diarrheal patients (China in 2014) [16]; ST163 and ST169 contained isolates from water (Canada in 2015); ST161 contained isolates from water (Canada in 2015) and a diarrheal patient (China in 2015) [16]; ST85, ST183, ST214, ST216, ST217, ST225, and ST237 each contained isolates from diarrheal patient in our previous studies [10,18]; ST187 and ST219 each contained isolates from healthy individuals in our previous study [16]; ST258 and ST260 each contained isolates from the Netherlands. Our analysis by clonal complexes which allowed one to examine closely related STs, found 17 C. freundii CCs containing at least 2 STs per CC contained isolates from different countries with one CC present in 5 different countries. For C. youngae and C. braakii, fewer CCs were identified, and narrower geographic distribution was found. However, there was no information from other countries for comparison for these latter two species. Thus, some Citrobacter STs and CCs are likely to be widely present in fecal, food, and other reservoirs and spread in different countries or regions. However, there are very limited reports of Citrobacter spp. from different sources or geographical regions. A recent study in companion animals reported nosocomial dissemination of C. freundii strains resistant to extended-spectrum cephalosporins [28]. Our studies underscore the need for further study to understand the genetic diversity, virulence and antibiotic resistance and their risks to human health.

\subsection{Association of C. freundii Lineage II and C. youngae Lineage V with Higher Adhesion and Cytotoxicity}

The clustering of highly adhesive and cytotoxic isolates in specific lineages is most interesting as it suggests that different lineages have different levels of virulence and/or may cause different types of diseases. Combining this study with our previous studies [10,16], there were nine highly cytotoxic C. freundii isolates, of which six were isolated from diarrheal patients, two from foods, and one from the environment. Among these six highly cytotoxic C. freundii isolates from diarrheal patients, five isolates were clustered in Lineage II and one in Lineage I, suggesting that Lineage II is more likely to cause diarrheal disease. C. freundii, as the most common Citrobacter species, has caused gastroenteritis associated outbreaks [3]. However, despite reports of different virulence factors involved [9,10], little is known of its pathogenic mechanisms and there have been no means that can differentiate strains that can cause diarrheal disease from those of harmless colonizers. The identification of Lineage II as more likely to cause diarrhea provided an avenue to further identify virulence genes involved and to determine whether Lineage II is more likely to cause other infections.

Similarly, combining with our previous studies [10,16], seven of the eight highly cytotoxic C. youngae strains fell into Lineage $\mathrm{V}$, of which four were isolated from diarrheal patients, suggest 
that Lineage $\mathrm{V}$ is likely to cause diarrheal disease. However, $C$. younage had not been recognized as a diarrheal pathogen and Lineage $\mathrm{V}$ should be further investigated for their potential to cause diarrheal disease.

C. braakii has been isolated from foods, hospital infections and UTIs [14], and acute peritonitis patients $[7,8]$. In our previous study, we isolated C. braakii isolates from food source, but not human fecal samples [10]. In this study, we obtained 45 C. braakii isolates from food, environment and diarrheal patients with two highly adhesive and five highly cytotoxic isolates. Three of the five highly cytotoxic C. braakii isolates were isolated from diarrheal patients, and it remains to be determined whether C. braakii can cause diarrhea.

\subsection{Higher Prevalence of Multidrug Resistance in C. braakii Isolates and Citrobacter Isolates from Food Sources}

MDR has been reported in Citrobacter isolates, especially C. freundii [17]. Considering isolates from this study only, 58 of the 128 isolates were MDR, mainly distributed among Lineages I to III. When combined with our previous studies [10,16], 96 of the 271 Citrobacter isolates $(35.4 \%)$ were MDR, including 58 C. freundii isolates ( $36.0 \%$ of C. freundii isolates, Lineages I and II), 12 C. youngae isolates ( $21.1 \%$ of $C$. youngae isolates, Lineages IV and V) and 26 C. braakii isolates $(49.0 \%$ of $C$. braakii isolates, Lineage III). The difference in the prevalence of MDR between $C$. youngae and C. braakii is statistically significant $(\mathrm{p}<0.01)$.

Among the 96 MDR isolates, 34 were isolated from diarrheal patients (25.8\% of diarrheal isolates), 50 from foods ( $47.6 \%$ of the food isolates), four from the environment $(36.4 \%)$ and eight from healthy individuals (34.8\%). Interestingly MDR were more prevalent among food isolates. Since most of the food source was related to meat or meat products $[10,16]$, the MDR may have been a result of extensive use of antibiotics in animals. Moreover, four highly cytotoxic strains (11.8\%) were found in 34 MDR isolates from diarrheal patients, highlighting the combined increased risk of high cytotoxicity and MDR of Citrobacter to human health.

\subsection{Carriage of ESBL Genes by Citrobacter spp. was Relatively Low}

ESBL producing Enterobacteriaceae has been a major challenge in infection control [29,30]. Previous studies have reported that Citrobacter isolated from human clinical infections that carried ESBL genes varied from $5.6 \%$ to $67.5 \%$, including $b l a_{\mathrm{TEM}-1}, b_{\mathrm{SHV}-12,},{ } a_{\mathrm{CTX}-\mathrm{M}-15}$ and $b l a_{\mathrm{CTX}-\mathrm{M}}[3,28]$. In our studies, including our previous studies $[10,16]$, we found $2.6 \%$ of Citrobacter isolates carried the bla ${ }_{\mathrm{CTX}-\mathrm{M}-9}$ gene, $1.1 \%$ carried the $b l a_{\mathrm{CTX}-\mathrm{M}-3}$ gene, $3.7 \%$ carried the $b l a_{\mathrm{TEM}-1}$ gene, and none carried the $b l a_{\mathrm{SHV}}$ gene. The ESBL carrying Citrobacter isolates consisted of 12 C. freundii isolates (7.5\% of $C$. freundii isolates) and four $C$. braakii isolates (7.5\% of $C$. braakii isolates). The four ESBL carrying C. braakii isolates were isolated from foods. Three of the 12 ESBLs carrying $C$. freundii isolates were isolated from diarrheal patients (2.3\% of diarrheal isolates), eight from foods $(7.6 \%)$ and one from healthy individuals (4.3\%). In comparison to other reports, ESBL carriage is lower in our isolates. The isolates of our previous two studies $[10,16]$ were from the south of China (Anhui province), while isolates of this study were from northern China (Hebei Province), suggesting likely low prevalence of ESBLs in Citrobacter spp. In China. However, other regions of China would need to be sampled to obtain a more comprehensive picture.

\subsection{Higher Prevalence of Quinolone Resistance in Lineages I and III of Citrobacter spp with Multiple Mechanism of Resistance Detected}

The prevalence of quinolone resistance varied among the lineages. Lineage III (C. braakii) had the highest proportion of resistance isolates $(52.6 \%)$, followed by Lineage I (C. freundii) with $23.7 \%$. We analyzed the carriage of potential quinolone resistance genes or mutations which include both the QRDR of gyrA and parC associated resistance, and PMQR genes mediated resistance [22]. Citrobacter isolates with mutations in the QRDR of gyrA, including Thr83Ile and Asp87Asn have shown reduced susceptibility to fluoroquinolones [22,23]. In our previous study [18], four (66.7\%) of the six 
ciprofloxacin-resistant $C$. freundii isolates were found to have mutations in codons 59,111 , and/or 134 in the QRDR region of the gyrA gene, namely, Thr59Ile, Gln111Arg, and Ile134Val. In this study, 28 ciprofloxacin-resistant isolates carried mutations in the QRDR of gyrA with 27 having one mutation, Thr59Ile and one having three mutations, Thr59Ile, Gln111Arg and Ile134Val. However, it should be noted that these three mutations have not been experimentally confirmed whether they affect fluoroquinolone resistance.

For PMQR gene-mediated resistance, $14.0 \%$ of our Citrobacter isolates carried a qnr gene, and $5.5 \%$ of Citrobacter isolates carried the aac $\left(6^{\prime}\right)-\mathrm{Ib}$-cr gene. Our results contrast previous reports of high prevalence of $q n r$ and $a a c\left(6^{\prime}\right)-I b-c r$ genes from clinical infections in China at $72.8 \%$ and $68.9 \%$ in C. freundii isolates; and $42.9 \%$ and $42.9 \%$ in C. braakii isolates, respectively from the study of Zhang et al. [29], and 63.3\% and $26.7 \%$ in C. freundii from the study of Yang et al. [31]. This difference could be due to different regions of China or the sources of samples.

\subsection{Citrobacter spp. Carried Many Variants of the qnrB Gene with C. freundii Lineage I as the Main Reservoir}

$q n r B$ is known to be harbored by Citrobacter [26]. Surprisingly our Citrobacter isolates harboured many $q n r B$ variants with 11 qnrB allelic variants found, including qnrB2, qnrB9, qnrB13, qnrB16, qnrB17, qnrB63, qnrB76, qnrB77, qnrB92, qnrB93 and qnrB94. Interestingly, although only $17 \%$ (28 of 161) of the $C$. freundii isolates harbored $q n r B$ genes, all except one $q n r B$ positive isolate belonged to Lineage I, suggesting that Lineage I is a main reservoir of $q n r B$ genes. However, carriage of $q n r B$ gene does not always confer high level of quinolone resistance [29]. Only two qnrB9-carrying $C$. freundii isolates in Lineage I were resistant to quinolones, while other $q n r B$-carrying $C$. freundii isolates in Lineage I were susceptible to NAL, CIP and LEV. The only q $n r B$ carrying Lineage II isolate is quinolones resistant. Four qnrB-carrying C. braakii isolates were resistant to NAL (MICs $\geq 128 \mu \mathrm{g} / \mathrm{mL}$ ). These results suggest that Citrobacter isolates carrying different $q n r B$ alleles may have different levels of quinolone resistance.

QnrB has a growing number of allelic variants [32]. In our previous study, we found a variant of qnrB77 (a qnrB77 contained a LexA binding site) and a new qnrB allele (qnrB92) $[10,16]$. In this study, we found a variant of $q n r B 76$ (a qnrB76 contained a LexA binding site), a variant of $q n r B 13$ (a qnrB13 contained a LexA binding site), and two new qnrB alleles, qnrB93 and qnrB94.

\section{Conclusions}

We analyzed 128 Citrobacter isolates (67 C. freundii, 16 C. youngae and 45 C. braakii isolates) from human diarrheal patients, foods and environment in Shijiazhuang, Hebei Province in the north of China. This study expands the genetic diversity observed from our previous studies of human and food isolates from South China $[10,16]$. The isolates showed high diversity with 123 STs of which 101 were novel STs. Only 22 STs (17.9\%) were found from different sources and/or geographic regions. Phylogenetic analysis divided the 128 isolates into five lineages. Lineages I and II contained C. freundii isolates, while Lineages IV and V contained C. youngae isolates and Lineage III contained all C. braakii isolates.

The 51 MDR isolates were mainly distributed in Lineage I (C. freundii) and Lineage III (C. braakii) with $48.3 \%$ and $46.7 \%$ of the isolates in these lineages being MDR, respectively. Food isolates were also more likely to be MDR. Combining data with our previous studies $[10,16]$, we found that the prevalence of quinolone resistance was highest in Lineage I (C. freundii) and Lineage III (C. braakii). Citrobacter spp. carried many variants of the qnrB gene with the carriage by $C$. freundii Lineage I isolates being the highest. Surprisingly, the prevalence of ESBL genes in Citrobacter spp. is relatively low.

There were 22 highly cytotoxic Citrobacter isolates, which were preferentially distributed in C. freundii Lineage II and C. youngae Lineage V, suggesting that these two lineages are more virulent than others, and thus, more likely to cause disease. This study has shed more light on the genetic diversity, pathogenicity and antibiotic resistance of Citrobacter. 


\section{Methods}

\subsection{Citrobacter Isolates}

128 Citrobacter spp. isolates were isolated from 50 diarrheal patients, 11 environment and 67 food samples in Shijiazhuang Hebei Province, China from 2016 to 2017. 50 diarrheal patient fecal samples included 30 C. freundii, eight $C$. braakii and 12 C. youngae isolates, and four of the 50 diarrheal patient fecal samples harbored norovirus (HB2016002 and HB2017022) or Vibrio parahaernolyticus (HB2016004 and HB2016036); the 11 environment isolates had seven $C$. freundii and four $C$. braakii which were isolated from food processing place; 67 food isolates included 30 C. freundii, 33 C. braakii and four C. youngae isolates which were isolated from chicken, pork, duck and vegetables (Supplementary Table S4). The species identity of each isolate was confirmed using API 20E test strips (bioMérieux, La Balme les Grottes, France). Bacterial culture media and conditions were as previously described [10].

\subsection{MLST and Phylogenetic Analysis}

The Citrobacter MLST scheme (http://pubmlst.org/cfreundii/) was used. PCR amplification and sequencing were carried out using published primers as described previously [10]. SeqMan 7.0 software was used to analyze the sequences.

\subsection{Antimicrobial Susceptibility Testing}

Antimicrobial susceptibility testing was carried out as previously described [10,33]. Minimum inhibitory concentration (MIC) was interpreted in 22 antibiotics, including ampicillin, cefotaxime, ceftazidime, cefepime, cefoxitin, ceftiofur Sodium, aztreonam, imipenem, meropenem, nalidixicacid, ciprofloxacin, levofloxacin, gentamicin, amikacin, streptomycin, kanamycin, tetracycline, doxycycline, chloramphenicol, trimethoprim/sulfamethoxazole, sulfafurazole and azithromycin (Table 2). E. coli ATCC 25922 and Pseudomonas aeruginosa ATCC 27853 were used as quality controls for MICs.

\subsection{PCR Amplification and Sequencing.}

Detection of $b l a_{\mathrm{TEM}}, b l a_{\mathrm{SHV}}, b l a_{\mathrm{CTX}-\mathrm{M}-1}, b l a_{\mathrm{CTX}-\mathrm{M}-2}, b l a_{\mathrm{CTX}-\mathrm{M}-8}, b l a_{\mathrm{CTX}-\mathrm{M}-9}, b l a_{\mathrm{GES}}, b l a_{\mathrm{PER}}$ and $b l a_{\mathrm{VEB}}, q n r A, q n r B, q n r S, q n r C, q n r D, a a c\left(6^{\prime}\right)-I b-c r$ and $q e p A$ genes were carried out as previously described [10,34-39].

\subsection{In Vitro Adhesion and Cytotoxicity Assays.}

The human epidermoid laryngocarcinoma (HEp-2, CCC0068) cell line was obtained from the cell resource center at Peking Union Medical College. HEp-2 cell line has been used as a human cell line to study bacteria-host interactions in many studies [40-42]. Note that Hep-2 cell line may have been a misidentified cell line, raising concerns of relevance to laryngeal cancer research [43], but the issue is not relevant here. In vitro adhesion to host cells was performed as previously described [10]. The mean number of bacteria per HEp-2 after examination of 10 visual fields was determined and was used as an adhesion index $(<1 ;>1$ and $<50 ;>50)$ [10].

The lactate dehydrogenase (LDH) released by the HEp-2 cells was determined as previously described [10]. All experiments were performed three times in duplicate.

\subsection{Statistical Analysis.}

SPSS software version 13.0 (SPSS Inc., Chicago, IL, USA) was used to conduct all statistical comparisons. A nonparametric test (Mann-Whitney U-test) and chi-square test were used to compare the different groups for statistical significance. $p$-value of $\leq 0.05$ (2-tailed) was considered to be statistically significant. 


\subsection{Ethics Approval and Consent to Participate}

This study was reviewed and approved by the ethics committee of the National Institute for Communicable Disease Control and Prevention, the Chinese CDC. Human fecal specimens were acquired with the written informed consent of the diarrheal patients with the approval of the ethics committee of the National Institute for Communicable Disease Control and Prevention, according to the medical research regulations of Ministry of Health (ICDC-2016004).

Supplementary Materials: The following are available online at http://www.mdpi.com/2076-0817/9/3/195/s1, Figure S1, Phylogenetic relationships as determined by MLST data for the $123 \mathrm{C}$. freundii isolates from this study and two previous studies; Table S1, List of clonal complexes of Citrobacter isolates from this study and the public MLST database ${ }^{\#}$; Table S2, Prevalence of MDR isolates in different Citrobacter species; Table S3, Adherence and Cytotoxicity in 128 Citrobacter Isolates; Table S4, Source, drugs resistance, Genotypes and Antibiotic resistance phenotype of 128 Citrobacter Isolates.

Author Contributions: Formal Analysis, L.Q., S.H., X.L., X.C., C.Z. and H.S.; Resources, B.X., Y.G. and R.J.; Writing-Original Draft Preparation, L.L.; Writing-review and editing, L.L. and R.L.; Supervision, J.X. All authors have read and approved the final version of the manuscript.

Funding: This research was funded by the National Natural Science Foundation of China (No. 81301401, 81290345), and the National Key Research and Development Program of China (2016YFC1201903, 2016YFC1202700).

Conflicts of Interest: The authors declare no conflict of interest.

\section{References}

1. Park, Y.-J.; Yu, J.K.; Lee, S.; Oh, E.J.; Woo, G.-J. Prevalence and diversity of qnr alleles in AmpC-producing Enterobacter cloacae, Enterobacter aerogenes, Citrobacter freundii and Serratia marcescens: A multicentre study from Korea. J. Antimicrob. Chemother. 2007, 60, 868-871. [CrossRef] [PubMed]

2. Guerrant, R.L.; Dickens, M.D.; Wenzel, R.P.; Kapikian, A.Z. Toxigenic bacterial diarrhea: Nursery outbreak involving multiple bacterial strains. J. Pediatr. 1976, 89, 885-891. [CrossRef]

3. Tschäpe, H.; Prager, R.; Streckel, W.; Fruth, A.; Tietze, E.; Böhme, G. Verotoxinogenic Citrobacter freundii associated with severe gastroenteritis and cases of haemolytic uraemic syndrome in a nursery school: Green butter as the infection source. Epidemiol. Infect. 1995, 114, 441-450. [CrossRef] [PubMed]

4. Warner, R.D. A large nontypical outbreak of Norwalk virus. Gastroenteritis associated with exposing celery to nonpotable water and with Citrobacter freundii. Arch. Intern. Med. 1991, 151, 2419-2424. [CrossRef] [PubMed]

5. Doulgeraki, A.; Paramithiotis, S.; Nychas, G.-J.E. Characterization of the Enterobacteriaceae community that developed during storage of minced beef under aerobic or modified atmosphere packaging conditions. Int. J. Food Microbiol. 2011, 145, 77-83. [CrossRef]

6. Giammanco, G.M.; Aleo, A.; Guida, I.; Mammina, C. Molecular Epidemiological Survey of Citrobacter freundii Misidentified as Cronobacter spp. (Enterobacter sakazakii) and Enterobacter hormaechei Isolated from Powdered Infant Milk Formula. Foodborne Pathog. Dis. 2011, 8, 517-525. [CrossRef]

7. Samonis, G.; Karageorgopoulos, D.; Kofteridis, D.P.; Matthaiou, D.; Sidiropoulou, V.; Maraki, S.; Falagas, M.E. Citrobacter infections in a general hospital: Characteristics and outcomes. Eur. J. Clin. Microbiol. Infect. Dis. 2008, 28, 61-68. [CrossRef]

8. Mohanty, S.; Singhal, R.; Sood, S.; Dhawan, B.; Kapil, A.; Das, B.K. Citrobacter infections in a tertiary care hospital in Northern India. J. Infect. 2007, 54, 58-64. [CrossRef]

9. Bai, L.; Xia, S.; Lan, R.; Liu, L.; Ye, C.; Wang, Y.; Jin, N.; Cui, Z.; Jing, H.; Xiong, Y.; et al. Isolation and Characterization of Cytotoxic, Aggregative Citrobacter freundii. PLoS ONE 2012, 7, e33054. [CrossRef]

10. Liu, L.; Lan, R.; Liu, L.; Wang, Y.; Zhang, Y.; Wang, Y.; Xu, J. Antimicrobial Resistance and Cytotoxicity of Citrobacter spp. in Maanshan Anhui Province, China. Front. Microbiol. 2017, 8, 1357. [CrossRef]

11. Chen, K.J.; Chen, T.H.; Sue, Y.M. Citrobacter Youngae and Pantoea Agglomerans Peritonitis in a Peritoneal Dialysis Patient. Perit. Dial. Int. 2013, 33, 336-337. [CrossRef] [PubMed]

12. Basra, P.; Koziol, A.; Wong, A.; Carrillo, C. Complete Genome Sequences of Citrobacter braakii Strains GTA-CB01 and GTA-CB04, Isolated from Ground Beef. Genome Announc. 2015, 3, e1307-14. [CrossRef] [PubMed] 
13. Kwak, H.L.; Han, S.K.; Park, S.; Park, S.H.; Shim, J.Y.; Oh, M.; Ricke, S.; Kim, H.Y. Development of a rapid and accurate identification method for Citrobacter species isolated from pork products using a Matrix-Assisted Laser-Desorption Ionization Time-of-Flight Mass Spectrometry (MALDI-TOFMS). J. Microbiol. Biotechnol. 2015, 25, 1537-1541. [CrossRef] [PubMed]

14. Arens, S.; Verhaegen, J.; Verbist, L. Differentiation and susceptibility of Citrobacter isolates from patients in a university hospital. Clin. Microbiol. Infect. 1997, 3, 53-57. [CrossRef]

15. Chao, C.-T.; Lee, S.-Y.; Yang, W.-S.; Chen, H.-W.; Fang, C.-C.; Yen, C.-J.; Chiang, C.-K.; Hung, K.-Y.; Huang, J.-W. Citrobacter Peritoneal Dialysis Peritonitis: Rare Occurrence with Poor Outcomes. Int. J. Med Sci. 2013, 10, 1092-1098. [CrossRef]

16. Liu, L.; Chen, D.; Liu, L.; Lan, R.; Hao, S.; Jin, W.; Sun, H.; Wang, Y.; Liang, Y.; Xu, J. Genetic Diversity, Multidrug Resistance, and Virulence of Citrobacter freundii From Diarrheal Patients and Healthy Individuals. Front. Microbiol. 2018, 8, 233. [CrossRef]

17. Akya, A.; Jafari, S.; Ahmadi, K.; Elahi, A. Frequency Of blaCTX-M, blaTEM and blaSHV Genes in Citrobacters Isolated from Imam Reza Hospital in Kermanshah. J. Mazand. Univ. Med. Sci. 2015, 25, 65-73.

18. Oliveira, H.; Pinto, G.; Oliveira, A.; Oliveira, C.; Faustino, M.A.; Briers, Y.; Domingues, L.; Azeredo, J. Characterization and genome sequencing of a Citrobacter freundii phage CfP1 harboring a lysin active against multidrug-resistant isolates. Appl. Microbiol. Biotechnol. 2016, 100, 10543-10553. [CrossRef]

19. Moland, E.S.; Hanson, N.D.; Black, J.A.; Hossain, A.; Song, W.; Thomson, K.S. Prevalence of Newer $\beta$-Lactamases in Gram-Negative Clinical Isolates Collected in the United States from 2001 to 2002. J. Clin. Microbiol. 2006, 44, 3318-3324. [CrossRef]

20. Choi, S.-H.; Lee, J.E.; Park, S.J.; Kim, M.-N.; Choo, E.J.; Kwak, Y.G.; Jeong, J.-Y.; Woo, J.H.; Kim, N.J.; Kim, Y.S. Prevalence, microbiology, and clinical characteristics of extended-spectrum $\beta$-lactamase-producing Enterobacter spp., Serratia marcescens, Citrobacter freundii, and Morganella morganii in Korea. Eur. J. Clin. Microbiol. Infect. Dis. 2007, 26, 557-561. [CrossRef]

21. Shao, Y.; Xiong, Z.; Li, X.; Hu, L.; Shen, J.; Li, T.; Hu, F.; Chen, S. Prevalence of plasmid-mediated quinolone resistance determinants in Citrobacter freundii isolates from Anhui province, PR China. J. Med Microbiol. 2011, 60, 1801-1805. [CrossRef] [PubMed]

22. Minarini, L.; Darini, A.L.C. Mutations in the quinolone resistance-determining regions of gyrA and parC in Enterobacteriaceae isolates from Brazil. Braz. J. Microbiol. 2012, 43, 1309-1314. [CrossRef] [PubMed]

23. Weigel, L.M.; Steward, C.D.; Tenover, F.C. gyrA Mutations Associated with Fluoroquinolone Resistance in Eight Species of Enterobacteriaceae. Antimicrob. Agents Chemother. 1998, 42, 2661-2667. [CrossRef] [PubMed]

24. Feil, E.J. Small change: Keeping pace with microevolution. Nat. Rev. Genet. 2004, 2, 483-495. [CrossRef] [PubMed]

25. Wirth, T.; Falush, D.; Lan, R.; Colles, F.; Mensa, P.; Wieler, L.H.; Karch, H.; Reeves, P.; Maiden, M.C.; Ochman, H.; et al. Sex and virulence in Escherichia coli: An evolutionary perspective. Mol. Microbiol. 2006, 60, 1136-1151. [CrossRef]

26. Jacoby, G.; Cattoir, V.; Hooper, D.; Martínez-Martínez, L.; Nordmann, P.; Pascual, A.; Poirel, L.; Wang, M. qnr Gene Nomenclature. Antimicrob. Agents Chemother. 2008, 52, 2297-2299. [CrossRef]

27. Liu, X.; Huang, Y.; Xu, X.; Zhao, Y.; Sun, Q.; Zhang, Z.; Zhang, X.; Wu, Y.; Wang, J.; Zhou, N.; et al. Complete Genome Sequence of Multidrug-Resistant Citrobacter freundii Strain P10159, Isolated from Urine Samples from a Patient with Esophageal Carcinoma. Genome Announc. 2016, 4, e01754-15. [CrossRef]

28. Harada, K.; Shimizu, T.; Ozaki, H.; Kimura, Y.; Miyamoto, T.; Tsuyuki, Y. Characterization of Antimicrobial Resistance in Serratia spp. and Citrobacter spp. Isolates from Companion Animals in Japan: Nosocomial Dissemination of Extended-Spectrum Cephalosporin-Resistant Citrobacter freundii. Microorganisms 2019, 7, 64. [CrossRef]

29. Liu, L.-H.; Wang, N.-Y.; Wu, A.Y.-J.; Lin, C.-C.; Lee, C.-M.; Liu, C.-P. Citrobacter freundii bacteremia: Risk factors of mortality and prevalence of resistance genes. J. Microbiol. Immunol. Infect. 2018, 51, 565-572. [CrossRef]

30. Zhang, R.; Ichijo, T.; Huang, Y.-L.; Cai, J.; Zhou, H.; Yamaguchi, N.; Nasu, M.; Chen, G.-X. High prevalence of qnr and aac $\left(6^{\prime}\right)$-Ib-cr genes in both water-borne environmental bacteria and clinical isolates of Citrobacter freundii in China. Microbes Environ. 2012, 27, 158-163. [CrossRef] 
31. Yang, H.; Chen, H.; Yang, Q.; Chen, M.; Wang, H. High Prevalence of Plasmid-Mediated Quinolone Resistance Genes qnr and aac(6 $\left.6^{\prime}\right)-\mathrm{Ib}-\mathrm{cr}$ in Clinical Isolates of Enterobacteriaceae from Nine Teaching Hospitals in China. Antimicrob. Agents Chemother. 2008, 52, 4268-4273. [CrossRef] [PubMed]

32. Jacoby, G.A.; Griffin, C.M.; Hooper, D.C. Citrobacter spp. as a Source of qnrB Alleles. Antimicrob. Agents Chemother. 2011, 55, 4979-4984. [CrossRef] [PubMed]

33. Clinical and Laboratory Standards Institute. M100-S28 Performance Standards for Antimicrobial Susceptibility Testing: Twenty-Eighth Informational Supplement; Clinical and Laboratory Standards Institute: Wayne, PA, USA, 2018; p. 353.

34. Poirel, L.; Walsh, T.; Cuvillier, V.; Nordmann, P. Multiplex PCR for detection of acquired carbapenemase genes. Diagn. Microbiol. Infect. Dis. 2011, 70, 119-123. [CrossRef] [PubMed]

35. Voets, G.M.; Fluit, A.C.; Scharringa, J.; Stuart, J.C.; Hall, M.A.L.-V. A set of multiplex PCRs for genotypic detection of extended-spectrum $\beta$-lactamases, carbapenemases, plasmid-mediated AmpC $\beta$-lactamases and OXA $\beta$-lactamases. Int. J. Antimicrob. Agents 2011, 37, 356-359. [CrossRef] [PubMed]

36. Liu, Y.-Y.; Wang, Y.; Walsh, T.; Yi, L.-X.; Zhang, R.; Spencer, J.; Doi, Y.; Tian, G.; Dong, B.; Huang, X.; et al. Emergence of plasmid-mediated colistin resistance mechanism MCR-1 in animals and human beings in China: A microbiological and molecular biological study. Lancet Infect. Dis. 2016, 16, 161-168. [CrossRef]

37. Xavier, B.B.; Lammens, C.; Ruhal, R.; Kumar-Singh, S.; Butaye, P.; Goossens, H.; Malhotra-Kumar, S. Identification of a novel plasmid-mediated colistin-resistance gene,mcr-2, inEscherichia coli, Belgium, June 2016. Eurosurveillance 2016, 21, 30280. [CrossRef]

38. Zhao, W.-H.; Hu, Z.-Q. IMP-type metallo-?-lactamases in Gram-negative bacilli: Distribution, phylogeny, and association with integrons. Crit. Rev. Microbiol. 2011, 37, 214-226. [CrossRef]

39. Frank, J.A.; Reich, C.I.; Sharma, S.; Weisbaum, J.S.; Wilson, B.A.; Olsen, G.J. Critical Evaluation of Two Primers Commonly Used for Amplification of Bacterial 16S rRNA Genes. Appl. Environ. Microbiol. 2008, 74, 2461-2470. [CrossRef]

40. Mange, J.-P.; Stephan, R.; Borel, N.; Wild, P.; Kim, K.S.; Pospischil, A.; Lehner, A. Adhesive properties of Enterobacter sakazakii to human epithelial and brain microvascular endothelial cells. BMC Microbiol. 2006, 6, 58. [CrossRef]

41. Dallal, M.M.S.; Validi, M.; Douraghi, M.; Fallah-Mehrabadi, J.; Lormohammadi, L. Evaluation the cytotoxic effect of cytotoxin-producing Klebsiella oxytoca isolates on the HEp-2 cell line by MTT assay. Microb. Pathog. 2017, 113, 416-420. [CrossRef]

42. Konkel, M.E.; Joens, L.A. Adhesion to and invasion of HEp-2 cells by Campylobacter spp. Infect. Immun. 1989, 57, 2984-2990. [CrossRef] [PubMed]

43. Philippe, G. A comprehensive review of Hep-2 cell line in translational research for laryngeal cancer. Am. J. Cancer Res. 2019, 9, 644-649.

(C) 2020 by the authors. Licensee MDPI, Basel, Switzerland. This article is an open access article distributed under the terms and conditions of the Creative Commons Attribution (CC BY) license (http://creativecommons.org/licenses/by/4.0/). 